\title{
Higgs decays to $Z Z$ and $Z \gamma$ in the standard model effective field theory: An NLO analysis
}

\author{
S. Dawson and P. P. Giardino \\ Department of Physics, Brookhaven National Laboratory, Upton, New York 11973, USA
}

(Received 13 January 2018; published 15 May 2018)

\begin{abstract}
We calculate the complete one-loop electroweak corrections to the inclusive $H \rightarrow Z Z$ and $H \rightarrow Z \gamma$ decays in the dimension-6 extension of the Standard Model Effective Field Theory (SMEFT). The corrections to $H \rightarrow Z Z$ are computed for on-shell $Z$ bosons and are a precursor to the physical $H \rightarrow Z f \bar{f}$ calculation. We present compact numerical formulas for our results and demonstrate that the logarithmic contributions that result from the renormalization group evolution of the SMEFT coefficients are larger than the finite next-to-leading-order contributions to the decay widths. As a byproduct of our calculation, we obtain the first complete result for the finite corrections to $G_{\mu}$ in the SMEFT.
\end{abstract}

DOI: $10.1103 /$ PhysRevD.97.093003

\section{INTRODUCTION}

The LHC experimental discovery of the Higgs boson, along with the measurement of Higgs properties that are in rough agreement with the Standard Model (SM) predictions, suggests that the SM is a valid effective theory at the weak scale. The lack of new particles up to the TeV scale makes possible the parametrization of possible high scale physics effects in terms of higher-dimension operators containing only SM fields [1]. In this paper, we study new physics contributions to Higgs decays in the context of the dimension-6 Standard Model Effective Field Theory (SMEFT). When compared with precise theoretical calculations, measurements of Higgs properties serve to constrain the coefficients of the higher-dimension operators and restrict possible beyond the SM (BSM) physics at energies $\Lambda \gg v$.

We study Higgs decays to $Z$ boson pairs and to $Z \gamma$ in the context of the SMEFT, where new physics is described by a tower of operators,

$$
\mathcal{L}=\mathcal{L}_{\mathrm{SM}}+\Sigma_{k=5}^{\infty} \Sigma_{i=1}^{n} \frac{\mathcal{C}_{i}^{k}}{\Lambda^{k-4}} O_{i}^{k}
$$

The dimension- $k$ operators are constructed from SM fields, and the BSM physics effects reside in the coefficient functions, $C_{i}^{k}$. For large $\Lambda$, it is sufficient to retain only the lowest-dimensional operators. We assume lepton number conservation, so the lowest-dimension relevant operators are dimension 6. Ignoring flavor, there are 59

Published by the American Physical Society under the terms of the Creative Commons Attribution 4.0 International license. Further distribution of this work must maintain attribution to the author(s) and the published article's title, journal citation, and DOI. Funded by SCOAP ${ }^{3}$. dimension-6 operators that are $S U(3) \times S U(2) \times U(1)$ invariant combinations of the SM fields [1,2]. The operators have been classified in several different bases, which are related by the equations of motion [1-4]. In this paper, we will use the Warsaw basis of Ref. [2].

A detailed understanding of Higgs properties requires the inclusion of the dimension-6 tree-level SMEFT effects, along with radiative corrections in the effective field theory. Measurements at the Higgs mass scale, $M_{H}$, that are sensitive to a set of SMEFT coefficients, $\mathcal{C}_{i}\left(M_{H}\right)$, at leading order will develop logarithmic sensitivity to other coefficients when the renormalization group evolved to the scale $\Lambda$, due to the renormalization group mixing of the coefficients [5-9]. There are also finite contributions that may be of the same numerical size as the logarithmic terms.

We compute the one-loop electroweak SMEFT contributions to the decays $H \rightarrow Z Z$ and $H \rightarrow Z \gamma$. These corrections include the one-loop SM electroweak corrections, along with the one-loop corrections due to the SMEFT operators of Eq. (1). Our results are interesting from a purely theoretical perspective, and we present the first complete one-loop SMEFT renormalization of $G_{\mu}$. The one-loop SMEFT corrections to $H \rightarrow b \bar{b}[10,11]$ and $H \rightarrow$ $\gamma \gamma[12,13]$ are known, as well as a general next-to-leadingorder (NLO) SMEFT calculation of two-body Higgs decays [14]. The physical process for $M_{H}=125 \mathrm{TeV}$ is $H \rightarrow Z f \bar{f}$, and our calculation is a precursor to the eventual one-loop three-body SMEFT calculation.

We review the SMEFT framework in Sec. II and our renormalization framework in Sec. III. Section IV contains numerical results for $H \rightarrow Z Z$ and $H \rightarrow Z \gamma$ and a discussion of the phenomenological impact of our calculation. A comparison with the physical off-shell process, $H \rightarrow Z f \bar{f}$, is in Appendix A. Appendix B contains numerical fits for the 
one-loop SMEFT result for $H \rightarrow Z Z$, and Appendix $C$ has analytic formulas for the logarithmic contributions to the one-loop SMEFT result for $H \rightarrow Z Z$. Lastly, Appendix D contains the one-loop SMEFT calculation of $G_{\mu}$.

\section{SMEFT BASICS}

In this section, we briefly introduce the SMEFT. We consider the Lagrangian in Eq. (1) truncated at dimension 6,

$$
\mathcal{L}=\mathcal{L}_{\mathrm{SM}}+\mathcal{L}_{\mathrm{EFT}}^{(6)} ; \quad \mathcal{L}_{\mathrm{EFT}}^{(6)}=\sum_{i} \frac{\mathcal{C}_{i}^{(6)}}{\Lambda^{2}} \mathcal{O}_{i}^{(6)},
$$

where $\mathcal{L}_{\mathrm{SM}}$ is the Standard Model Lagrangian and $\mathcal{L}_{\mathrm{EFT}}^{(6)}$ is the most general $S U(3) \times S U(2) \times U(1)$ invariant effective field theory (EFT) Lagrangian that can be built using only dimension-6 operators. In the following, we drop the superscript (6). Only a few operators contribute to the $H \rightarrow$ $Z Z$ and $H \rightarrow Z \gamma$ decays at tree level, while more operators contribute at one loop. In total, 19 of the 59 independent dimension- 6 operators of the Warsaw basis enter our calculation,

$$
\begin{aligned}
& \mathcal{O}_{W}, \mathcal{O}_{\phi}, \mathcal{O}_{\phi \square}, \mathcal{O}_{\phi D}, \mathcal{O}_{u \phi}, \mathcal{O}_{\phi W}, \mathcal{O}_{\phi B}, \mathcal{O}_{\phi W B}, \mathcal{O}_{u W}, \\
& \mathcal{O}_{u B}, \mathcal{O}_{\phi l}^{(1)}, \mathcal{O}_{\phi l}^{(3)}, \mathcal{O}_{\phi e}, \mathcal{O}_{\phi q}^{(1)}, \mathcal{O}_{\phi q}^{(3)}, \mathcal{O}_{\phi u}, \mathcal{O}_{\phi d}, \mathcal{O}_{l l}, \mathcal{O}_{l q}^{(3)},
\end{aligned}
$$

where the operators are defined in Table I,

$$
D_{\mu}=\partial_{\mu}+i g^{\prime} B_{\mu} Y+i g \frac{\tau^{I}}{2} W_{\mu}^{I}+i g_{s} T^{A} G_{\mu}^{A},
$$

$\tau^{I}$ are the Pauli matrices, and $l_{L}$ and $q_{L}$ are the $S U(2)_{L}$ doublet lepton and quark fields. For simplicity, we assume a diagonal flavor structure for the coefficients $\mathcal{C}$, i.e., $\mathcal{C}_{p, r}=\mathcal{C}_{i} \mathbf{1}_{p, r}$, where $p$ and $r$ are flavor indices.
Furthermore, we assume $\underset{e, \mu, \mu, e}{\mathcal{C}_{l l}}=\underset{\mu, e, e \mu}{\mathcal{C}_{l l}} \equiv \mathcal{C}_{l l} \quad$ and $\mathcal{C}_{l q}^{(3)}=\mathcal{C}_{l q}^{(3)} \equiv \mathcal{C}_{l q}^{(3)}$.

In general, the presence of the dimension- 6 operators changes the structure of the Lagrangian and the correlations between the Lagrangian quantities and the measured observables $[15,16]$. In the following, we discuss these modifications, as they are relevant to the one-loop SMEFT calculations of $H \rightarrow Z Z$ and $H \rightarrow Z \gamma$.

\section{A. Higgs sector}

In the SMEFT, the Higgs Lagrangian takes the form

$$
\begin{aligned}
\mathcal{L}= & \left(D^{\mu} \phi\right)^{\dagger}\left(D_{\mu} \phi\right)+\mu^{2} \phi^{\dagger} \phi-\lambda\left(\phi^{\dagger} \phi\right)^{2} \\
& +\frac{1}{\Lambda^{2}}\left(\mathcal{C}_{\phi}\left(\phi^{\dagger} \phi\right)^{3}+\mathcal{C}_{\phi \square}\left(\phi^{\dagger} \phi\right) \square\left(\phi^{\dagger} \phi\right)\right. \\
& \left.+\mathcal{C}_{\phi D}\left(\phi^{\dagger} D^{\mu} \phi\right)^{*}\left(\phi^{\dagger} D_{\mu} \phi\right)\right),
\end{aligned}
$$

where $\phi$ is the Higgs doublet

$$
\phi=\left(\begin{array}{c}
\phi^{+} \\
\frac{1}{\sqrt{2}}\left(v+H+i \phi^{0}\right)
\end{array}\right)
$$

and $v$ is the vacuum expectation value (vev) defined as the minimum of the potential,

$$
v \equiv \sqrt{2}\langle\phi\rangle=\sqrt{\frac{\mu^{2}}{\lambda}}+\frac{3 \mu^{3}}{8 \lambda^{5 / 2}} \frac{\mathcal{C}_{\phi}}{\Lambda^{2}} .
$$

\begin{tabular}{|c|c|c|c|c|c|}
\hline $\mathcal{O}_{W}$ & $\epsilon^{I J K} W_{\mu}^{I \nu} W_{\nu}^{J \rho} W_{\rho}^{K \mu}$ & $\mathcal{O}_{\phi}$ & $\left(\phi^{\dagger} \phi\right)^{3}$ & $\mathcal{O}_{\phi \square}$ & $\left(\phi^{\dagger} \phi\right) \square\left(\phi^{\dagger} \phi\right)$ \\
\hline $\mathcal{O}_{\phi D}$ & $\left(\phi^{\dagger} D^{\mu} \phi\right)^{*}\left(\phi^{\dagger} D_{\mu} \phi\right)$ & $\mathcal{O}_{p, r}$ & $\left(\phi^{\dagger} \phi\right)\left(\bar{q}_{p}^{\prime} u_{r}^{\prime} \tilde{\phi}\right)$ & $\mathcal{O}_{\phi W}$ & $\left(\phi^{\dagger} \phi\right) W_{\mu \nu} W^{\mu \nu}$ \\
\hline $\mathcal{O}_{\phi B}$ & $\left(\phi^{\dagger} \phi\right) B_{\mu \nu} B^{\mu \nu}$ & $\mathcal{O}_{\phi W B}$ & $\left(\phi^{\dagger} \tau^{I} \phi\right) W_{\mu \nu}^{I} B^{\mu \nu}$ & $\mathcal{O}_{u W}$ & $\left(\bar{q}_{p}^{\prime} \sigma^{\mu \nu} u_{r}^{\prime}\right) \tau^{I} \tilde{\phi} W_{\mu \nu}^{I}$ \\
\hline $\mathcal{O}_{p, r}$ & $\left(\bar{q}_{p}^{\prime} \sigma^{\mu \nu} u_{r}^{\prime}\right) \tilde{\phi} B_{\mu \nu}$ & $\mathcal{O}_{p l}^{(1)}$ & $\left(\phi^{\dagger} i \overleftrightarrow{D}_{\mu} \phi\right)\left(\bar{l}_{p}^{\prime} \gamma^{\mu} l_{r}^{\prime}\right)$ & $\underset{p, r}{\mathcal{O}_{\phi l}^{(3)}}$ & $\left(\phi^{\dagger} i \stackrel{\leftrightarrow}{D}_{\mu}^{I} \phi\right)\left(\bar{l}_{p}^{\prime} \tau^{I} \gamma^{\mu} l_{r}^{\prime}\right)$ \\
\hline $\mathcal{O}_{p e}$ & $\left(\phi^{\dagger} i \stackrel{\leftrightarrow}{D}_{\mu} \phi\right)\left(\bar{e}_{p}^{\prime} \gamma^{\mu} e_{r}^{\prime}\right)$ & $\mathcal{O}_{p q}^{(1)}$ & $\left(\phi^{\dagger} i \stackrel{\leftrightarrow}{D}_{\mu} \phi\right)\left(\bar{q}_{p}^{\prime} \gamma^{\mu} q_{r}^{\prime}\right)$ & $\mathcal{O}_{p q}^{(3)}$ & $\left(\phi^{\dagger} i \stackrel{\leftrightarrow}{D}_{\mu}^{I} \phi\right)\left(\bar{q}_{p}^{\prime} \tau^{I} \gamma^{\mu} q_{r}^{\prime}\right)$ \\
\hline $\begin{array}{c}\mathcal{O}_{\phi u} \\
p, r\end{array}$ & $\left(\phi^{\dagger} i \stackrel{\leftrightarrow}{D}_{\mu} \phi\right)\left(\bar{u}_{p}^{\prime} \gamma^{\mu} u_{r}^{\prime}\right)$ & $\mathcal{O}_{p, r}$ & $\left(\phi^{\dagger} i \stackrel{\leftrightarrow}{D}_{\mu} \phi\right)\left(\bar{d}_{p}^{\prime} \gamma^{\mu} d_{r}^{\prime}\right)$ & $\underset{p, r, s, t}{\mathcal{O}_{l l}}$ & $\left(\bar{l}_{p}^{\prime} \gamma_{\mu} l_{r}^{\prime}\right)\left(\bar{l}_{s}^{\prime} \gamma^{\mu} l_{t}^{\prime}\right)$ \\
\hline $\begin{array}{l}\mathcal{O}_{l q}^{(3)} \\
p, r, s, t\end{array}$ & $\left(\bar{l}_{p}^{\prime} \gamma_{\mu} \tau^{l} l_{r}^{\prime}\right)\left(\bar{q}_{s}^{\prime} \gamma^{\mu} \tau^{I} q_{t}^{\prime}\right)$ & & & & \\
\hline
\end{tabular}

Due to the presence of $\mathcal{O}_{\phi \square}$ and $\mathcal{O}_{\phi D}$ in Eq. (5), the kinetic terms in the resulting Lagrangian are not canonically normalized. As a consequence, we need to shift the fields,

TABLE I. Dimension-6 operators relevant for our study (from Ref. [2]). For brevity, we suppress fermion chiral indices $L$ and $R$. $I=1,2,3$ is an $S U(2)$ index, $p$ and $r$ are flavor indices, and $\phi^{\dagger} i \stackrel{\leftrightarrow}{D_{\mu}} \phi \equiv \phi^{\dagger} D_{\mu} \phi-\left(D_{\mu} \phi^{\dagger}\right) \phi$ 


$$
\begin{aligned}
H & \rightarrow H\left(1-\frac{v^{2}}{\Lambda^{2}}\left(\frac{1}{4} \mathcal{C}_{\phi D}-\mathcal{C}_{\phi \square}\right)\right), \\
\phi^{0} & \rightarrow \phi^{0}\left(1-\frac{v^{2}}{\Lambda^{2}}\left(\frac{1}{4} \mathcal{C}_{\phi D}\right)\right), \\
\phi^{+} & \rightarrow \phi^{+}
\end{aligned}
$$

and the physical mass of the Higgs, defined as the pole of the propagator, becomes

$$
M_{H}^{2}=2 \lambda v^{2}-\frac{v^{4}}{\Lambda^{2}}\left(3 \mathcal{C}_{\phi}-4 \lambda \mathcal{C}_{\phi \square}+\lambda C_{\phi D}\right) .
$$

As anticipated, the relation between the Lagrangian parameters and the measured observable $\left(M_{H}\right)$ is altered by the presence of the dimension- 6 operators $[15,16]$.

\section{B. Gauge sector}

The introduction of the operators in Eq. (3) also alters the form of the kinetic terms for the Lagrangian of the gauge sector. The relevant Lagrangian terms are

$$
\begin{aligned}
\mathcal{L}= & -\frac{1}{4} W I, \mu \nu W_{\mu \nu}^{I}-\frac{1}{4} B^{\mu \nu} B_{\mu \nu} \\
& +\frac{1}{\Lambda^{2}}\left(\mathcal{C}_{\phi W}\left(\phi^{\dagger} \phi\right) W^{I, \mu \nu} W_{\mu \nu}^{I}+\mathcal{C}_{\phi B}\left(\phi^{\dagger} \phi\right) B^{\mu \nu} B_{\mu \nu}\right. \\
& \left.+\mathcal{C}_{\phi W B}\left(\phi^{\dagger} \tau^{I} \phi\right) W^{I, \mu \nu} B_{\mu \nu}\right) .
\end{aligned}
$$

It is convenient to define "barred" fields, $\bar{W}_{\mu} \equiv(1-$ $\left.\mathcal{C}_{\phi W} v^{2} / \Lambda^{2}\right) W_{\mu}$ and $\bar{B}_{\mu} \equiv\left(1-\mathcal{C}_{\phi B} v^{2} / \Lambda^{2}\right) B_{\mu}$ and barred gauge couplings, $\bar{g}_{2} \equiv\left(1+\mathcal{C}_{\phi W} v^{2} / \Lambda^{2}\right) g_{2}$ and $\bar{g}_{1} \equiv(1+$ $\left.\mathcal{C}_{\phi B} v^{2} / \Lambda^{2}\right) g_{1}$, so that $\bar{W}_{\mu} \bar{g}_{2}=W_{\mu} g_{2}$ and $\bar{B}_{\mu} \bar{g}_{1}=B_{\mu} g_{1}$. The barred fields defined in this way have their kinetic terms properly normalized and preserve the form of the covariant derivative. The masses of the $\mathrm{W}$ and $\mathrm{Z}$ fields (poles of the propagators) are then expressed in terms of the barred couplings $[9,17]$ :

$$
\begin{aligned}
& M_{W}^{2}=\frac{\bar{g}_{2}^{2} v^{2}}{4}, \\
& M_{Z}^{2}=\frac{\left(\bar{g}_{1}^{2}+\bar{g}_{2}^{2}\right) v^{2}}{4}+\frac{v^{4}}{\Lambda^{2}}\left(\frac{1}{8}\left(\bar{g}_{1}^{2}+\bar{g}_{2}^{2}\right) \mathcal{C}_{\phi D}+\frac{1}{2} \bar{g}_{1} \bar{g}_{2} \mathcal{C}_{\phi W B}\right) .
\end{aligned}
$$

It is interesting to note that the extra terms in the definition of the $Z$ mass are due to the rotation, $\left(W_{\mu}^{3}, B_{\mu}\right) \rightarrow\left(Z_{\mu}, A_{\mu}\right)$, that is proportional to $\mathcal{C}_{\phi W B}$ and the shift of $\phi^{0}$ in Eq. (8) that is proportional to $\mathcal{C}_{\phi D}$.

\section{Fermion sector}

Lastly, we study the fermion sector. We notice that the presence of the dimension- 6 operators does not alter the kinetic terms in the Lagrangian, so we concentrate on the mass terms ${ }^{1}$ :

$$
\begin{aligned}
\mathcal{L}= & -\left(y_{e} \bar{l}_{L} e_{R} \phi+y_{u} \bar{q}_{L} u_{R} \tilde{\phi}+y_{d} \bar{q}_{L} d_{R} \phi+\text { H.c. }\right) \\
& +\frac{1}{\Lambda^{2}}\left(\mathcal{C}_{e \phi}\left(\phi^{\dagger} \phi\right)\left(\bar{l}_{L} e_{R} \phi\right)+\mathcal{C}_{u \phi}\left(\phi^{\dagger} \phi\right)\left(\bar{q}_{L} u_{R} \tilde{\phi}\right)\right. \\
& \left.+\mathcal{C}_{d \phi}\left(\phi^{\dagger} \phi\right)\left(\bar{q}_{L} d_{R} \phi\right)+\text { H.c. }\right) .
\end{aligned}
$$

The masses of all fermions are shifted by the interactions of Eq. (12). The lepton and light quark masses do not enter the one-loop result for $H \rightarrow Z Z$ and can be safely set to 0 there. However, the masses of the leptons and lighter quarks contribute logarithmically to the $\gamma \gamma$ wave function in the one-loop $H \rightarrow Z \gamma$ calculation, and we retain finite fermion masses there. However, since the lowest-order (LO) $H \rightarrow$ $Z \gamma$ amplitude is $\mathcal{O}\left(\frac{v^{2}}{\Lambda^{2}}\right)$, the contributions of the terms proportional to $\mathcal{C}_{e \phi}$ and $\mathcal{C}_{d \phi}$ to light fermion masses, $m_{f}$, are $\mathcal{O}\left(\frac{m_{f} v^{4}}{\Lambda^{4}}\right)$ and can be neglected. We concentrate on the definition of the top pole mass,

$$
M_{t}=\frac{v}{\sqrt{2}}\left(y_{t}-\frac{1}{2} \mathcal{C}_{u \phi} \frac{v^{2}}{\Lambda^{2}}\right) .
$$

Dimension-6 operators involving fermions also give contributions to the decay of the $\mu$ lepton, thus changing the relation between the vev, $v$, and the Fermi constant $G_{\mu}$ obtained from the measurement of the $\mu$ lifetime. Considering only contributions that interfere with the SM amplitude, we obtain the tree-level result,

$$
\begin{aligned}
G_{\mu} & =\frac{1}{\sqrt{2} v^{2}}-\frac{1}{2 \sqrt{2} \Lambda^{2}}\left(\underset{e, \mu, \mu, e}{\mathcal{C}_{l l}}+\underset{\mu, e, e \mu}{\mathcal{C}_{l l}}\right)+\frac{\sqrt{2}}{2 \Lambda^{2}}{\left(\mathcal{C}_{\phi l}{ }_{e, e}{ }^{(3)}+\underset{\mu, \mu}{\mathcal{C}_{\phi l}{ }^{(3)}}\right)} \equiv \frac{1}{\sqrt{2} v^{2}}-\frac{1}{\sqrt{2} \Lambda^{2}} \mathcal{C}_{l l}+\frac{\sqrt{2}}{\Lambda^{2}} \mathcal{C}_{\phi l}^{(3)},
\end{aligned}
$$

where in the last equality we assumed flavor universality of the coefficients.

\section{RENORMALIZATION}

The SM one-loop electroweak corrections to $H \rightarrow Z Z$ are well known, and we reproduce the results of Ref. [18]. The decay $H \rightarrow Z \gamma$ first occurs at one loop in the SM, and analytic results are in Refs. [19,20].

The calculations of the radiative corrections to $H \rightarrow Z Z$ and $H \rightarrow Z \gamma$ in the SMEFT proceed in the usual way [21] by the choice of a renormalization scheme, the definition of a suitable set of input parameters, and the calculation of the one-particle irreducible (1PI) amplitudes involved. However, since the SMEFT theory is only renormalizable order by order in the $\left(v^{2} / \Lambda^{2}\right)$ expansion, we need to add an

\footnotetext{
${ }^{1}$ We neglect flavor mixing, so $\mathcal{C}_{u \phi}$ represents generically $\mathcal{C}_{u \phi}$, $\mathcal{C}_{c \phi}, \mathcal{C}_{t \phi}$, etc.
} 
extra requirement and drop all terms proportional to $\left(v^{2} / \Lambda^{2}\right)^{a}$ with $a>1$. These terms would need counterterms of dimension 8 that are not included in our study. Dropping them makes it possible for us to proceed with our renormalization program. The one-loop SMEFT calculation contains both tree-level and one-loop contributions from the dimension- 6 operators, along with the full electroweak one-loop SM amplitude. We chose a modified on-shell (OS) scheme, where the SM parameters are OS quantities, while the SMEFT coefficients are defined as $\overline{\mathrm{MS}}$ quantities.

The tadpole counterterms are defined such that they cancel completely the tadpole graphs [22]. This condition forces us to identify the renormalized vacuum to be the minimum of the renormalized scalar potential at each order of perturbation theory. Notice that, due to this choice, the intermediate quantities defined here are gauge dependent, while the final result is gauge independent as expected.

We choose the $G_{\mu}$ scheme, where we take the physical input parameters to be ${ }^{2}$

$$
\begin{aligned}
G_{\mu} & =1.1663787(6) \times 10^{-5} \mathrm{GeV}^{-2} \\
M_{Z} & =91.1876 \pm .0021 \mathrm{GeV} \\
M_{W} & =80.385 \pm .015 \mathrm{GeV} \\
M_{H} & =125.09 \pm 0.21 \pm 0.11 \mathrm{GeV} \\
M_{t} & =173.1 \pm 0.6 \mathrm{GeV} .
\end{aligned}
$$

Since the coefficients of the dimension- 6 operators are not measured quantities, it is convenient to treat them as $\overline{\mathrm{MS}}$ parameters, so the renormalized coefficients are $\mathcal{C}(\mu)=$ $\mathcal{C}_{0}$ minus poles, where $\mathcal{C}_{0}$ are the bare quantities. The poles of the coefficients $\mathcal{C}_{0}$ are obtained from the renormalization group evolution of the coefficients computed in the unbroken phase of the theory in Refs. [7-9]. In general, one can write

$$
\mathcal{C}_{i}(\mu)=\mathcal{C}_{0, i}-\frac{1}{2 \hat{\epsilon}} \frac{1}{16 \pi^{2}} \gamma_{i j} \mathcal{C}_{j},
$$

where $\mu$ is the renormalization scale, $\gamma_{i j}$ is the one-loop anomalous dimension,

$$
\mu \frac{d \mathcal{C}_{i}}{d \mu}=\frac{1}{16 \pi^{2}} \gamma_{i j} \mathcal{C}_{j}
$$

and $\hat{\epsilon}^{-1} \equiv \epsilon^{-1}-\gamma_{E}+\log (4 \pi)$ is related to the regulator $\epsilon$ for integrals evaluated in $d=4-2 \epsilon$ dimensions.

\footnotetext{
${ }^{2}$ The light quark masses and lepton masses enter into the $\gamma$ wave function renormalization for $H \rightarrow Z \gamma$, and we take $m_{b}=$ $4.78 \mathrm{GeV}, \mathrm{m}_{c}=1.67 \mathrm{GeV}, \mathrm{m}_{\mathrm{s}}=0.1 \mathrm{GeV}, \mathrm{m}_{\mathrm{d}}=0.005 \mathrm{GeV}$, $\mathrm{m}_{\mathrm{u}}=0.002 \mathrm{GeV}, \quad \mathrm{m}_{\tau}=1.776 \mathrm{GeV}, \quad \mathrm{m}_{\mu}=0.105 \mathrm{GeV}, \quad$ and $\mathrm{m}_{e}=0.0005 \mathrm{GeV}$. The effects of the light fermions are small here, so a more sophisticated analysis is not warranted.
}

At one loop, the tree-level parameters of the previous section (denoted with the subscript 0 in this section) must be renormalized. The renormalized SM masses are defined by

$$
M_{V}^{2}=M_{0, V}^{2}-\Pi_{V}\left(M_{V}^{2}\right),
$$

where $\Pi_{V}\left(M_{V}^{2}\right)$ is the one-loop correction to the two-point function of either $\mathrm{Z}$ or $\mathrm{W}$ computed on shell. The gauge boson two-point functions in the SMEFT can be found in Refs. [14,23].

The one-loop relation between the vev and the Fermi constant is defined by the equation

$$
G_{\mu}+\frac{\mathcal{C}_{l l}}{\sqrt{2} \Lambda^{2}}-\sqrt{2} \frac{\mathcal{C}_{\phi l}^{(3)}}{\Lambda^{2}} \equiv \frac{1}{\sqrt{2} v_{0}^{2}}(1+\Delta r),
$$

where $v_{0}$ is the unrenormalized minimum of the potential and $\Delta r$ is obtained from the one-loop corrections to $\mu$ decay and is given by

$$
\begin{aligned}
\Delta r= & 2 v^{2} \mathcal{B}+\mathcal{V}\left(1+\frac{v^{2}}{\Lambda^{2}} \mathcal{C}_{\phi l}^{(3)}\right)+\mathcal{E}\left(1+\frac{v^{2}}{\Lambda^{2}}\left(2 \mathcal{C}_{\phi l}^{(3)}-\mathcal{C}_{l l}\right)\right) \\
& -\frac{A_{W W}}{M_{W}^{2}}\left(1+2 \frac{v^{2}}{\Lambda^{2}} \mathcal{C}_{\phi l}^{(3)}\right) \\
& +\frac{1}{16 \pi^{2}} \frac{1}{2 \hat{\epsilon}} \frac{v^{2}}{\Lambda^{2}}\left(2 \gamma_{\phi l, j}^{(3)} \mathcal{C}_{j}-\gamma_{l l, j} \mathcal{C}_{j}\right) .
\end{aligned}
$$

In Eq. (19), $\mathcal{B}$ is the box contribution, $\mathcal{V}$ is the vertex contribution, $A_{W W}=\Pi_{W}(0)$ is the W boson self-energy at zero momentum, and $\mathcal{E}$ is the sum of the lepton $\left(\mu, e, \nu_{\mu}, \nu_{e}\right)$ wave function renormalizations. All the quantities are calculated at zero external momenta. Notice that the definition of $\Delta r$ is modified with respect to the SM result due to the presence of dimension- 6 operators in the treelevel relation between $G_{\mu}$ and $v$ given in Eq. (18). Additionally, we absorb the poles of the coefficients $\mathcal{C}$ into the definition of $\Delta r$. The renormalization of the vev is then

$$
\begin{aligned}
v^{2} & =v_{0}^{2}-\delta v^{2} \\
\delta v^{2} & =\frac{\Delta r}{\sqrt{2} G_{\mu}}\left(1-\frac{1}{\sqrt{2} G_{\mu} \Lambda^{2}} \mathcal{C}_{l l}+\frac{\sqrt{2}}{G_{\mu} \Lambda^{2}} \mathcal{C}_{\phi l}^{(3)}\right) .
\end{aligned}
$$

Analytic expressions for $\Delta r$ in both the SM and the SMEFT at dimension 6 are given in Appendix $\mathrm{C}$ in the $R_{\xi}$.

In the following, we indicate with the symbol $\Delta \mathcal{A}^{\mu \nu}$ the sum of the contributions from the renormalization of the vev, the masses, and the SMEFT coefficients described above. The other contributions to the one-loop corrections are the proper 1PI amplitudes $\mathcal{A}_{1 P I}^{\mu \nu}$, the particle reducible contributions $\mathcal{A}_{P R}^{\mu \nu}$ due to the $Z / \gamma$ mixing which arises in the $H \rightarrow Z Z$ process, and the external leg wave function 
renormalization $\delta Z_{i}=-\partial \Pi_{i}\left(k^{2}\right) /\left.\partial k^{2}\right|_{M_{i}^{2}}$. The calculation of these contributions is relatively straightforward. We start with the $R_{\xi}$ Feynman rules for the SMEFT in the Warsaw basis presented in Ref. [17] and convert them to a FEYNARTS [24] model file, using the FEYnRules [25] routines, from which we obtain the amplitudes needed for our calculation. We reduce the integrals in terms of Passarino-Veltman integrals [26], using FeYNCALC [27,28], and lastly we use LoopTools [29] to numerically calculate the integrals. Once we compute all the terms that contribute, the one-loop correction can be simply written as

$$
\mathcal{A}^{1 l, \mu \nu}=\mathcal{A}_{1 P I}^{\mu \nu}+\mathcal{A}^{0 l, \mu \nu} \frac{1}{2} \sum_{i} \delta Z_{i}+\mathcal{A}_{P R}^{\mu \nu}+\Delta \mathcal{A}^{\mu \nu} .
$$

We verified that $\mathcal{A}^{1 l, \mu \nu}$ is UV and IR finite, and we confirmed its gauge invariance by computing the amplitudes for the $H \rightarrow Z Z$ and $H \rightarrow Z \gamma$ processes in the $R_{\xi}$ gauge.

We conclude this section with a few remarks on the truncation of the expansion in loops and powers of $\frac{v^{2}}{\Lambda^{2}}$. To clarify our explanation, we consider the generic form of the $H \rightarrow Z Z$ amplitude where we reintroduce the notation $\mathcal{C}^{(6)}$ and $\mathcal{C}^{(8)}$ for the coefficients of dimensions-6 and -8 operators:

$$
\begin{gathered}
\mathcal{A} \sim \hat{a}_{0} g_{\mathrm{SM}}+\hat{a}_{1} \mathcal{C}^{(6)} \frac{v^{2}}{\Lambda^{2}}+\hat{a}_{2} \frac{g_{\mathrm{SM}}^{3}}{16 \pi^{2}}+\hat{a}_{3} \mathcal{C}^{(6)} \frac{v^{2}}{\Lambda^{2}} \frac{g_{\mathrm{SM}}^{2}}{16 \pi^{2}} \\
+\frac{v^{4}}{\Lambda^{4}}\left(\hat{a}_{4}\left(\mathcal{C}^{(6)}\right)^{2} \frac{g_{\mathrm{SM}}}{16 \pi^{2}}+\hat{a}_{5} \mathcal{C}^{(8)}\right)+\ldots
\end{gathered}
$$

In Eq. (22), we assumed the ordering $g_{\mathrm{SM}}>\mathcal{C}^{(6)} \frac{v^{2}}{\Lambda^{2}}>$ $\frac{g_{\mathrm{SM}}^{3}}{16 \pi^{2}}>\mathcal{C}^{(8)} \sim\left(\mathcal{C}^{(6)}\right)^{2} \frac{g_{\mathrm{SM}}}{16 \pi^{2}}$, and we ordered the terms from largest to smallest according to it. Note that in Eq. (22) we have only included one insertion of dimension-6 operators at tree level, $\left(\hat{a}_{1}\right)$. If we were considering a more complicated process, it would in general be possible to have two (or more) tree-level insertions of dimension-6 operators, which would give a contribution of $\mathcal{O}\left(\frac{v^{4}}{\Lambda^{4}}\right)$ that would be of the same order as terms we have included. The squared amplitude then is

$$
\begin{aligned}
|\mathcal{A}|^{2} \sim & \hat{a}_{0}^{2} g_{\mathrm{SM}}^{2}+2 \hat{a}_{0} \hat{a}_{1} \mathcal{C}^{(6)} \frac{v^{2}}{\Lambda^{2}} g_{\mathrm{SM}}+\hat{a}_{1}^{2}\left(\mathcal{C}^{(6)}\right)^{2} \frac{v^{4}}{\Lambda^{4}} \\
& +2 \hat{a}_{0} \hat{a}_{2} \frac{g_{\mathrm{SM}}^{4}}{16 \pi^{2}}+2\left(\hat{a}_{1} \hat{a}_{2}+\hat{a}_{0} \hat{a}_{3}\right) \mathcal{C}^{(6)} \frac{v^{2}}{\Lambda^{2}} \frac{g_{\mathrm{SM}}^{3}}{16 \pi^{2}} \\
& +\frac{v^{4}}{\Lambda^{4}} g_{\mathrm{SM}}\left(2\left(\hat{a}_{0} \hat{a}_{4}+\hat{a}_{1} \hat{a}_{3}\right)\left(\mathcal{C}^{(6)}\right)^{2} \frac{g_{\mathrm{SM}}}{16 \pi^{2}}\right. \\
& \left.+\left(\hat{a}_{0} \hat{a}_{4}\right) \mathcal{C}^{(8)}\right)+\cdots
\end{aligned}
$$

As explained at the beginning of this section, in order to obtain a result that is finite in the UV in the dimension-6 SMEFT, we need to drop terms that are of order $\frac{v^{4}}{\Lambda^{4}}$ in the amplitude $\mathcal{A}$. However Eqs. (22) and (23) show that at the squared amplitude level the requirement is slightly different: while dropping the terms $\sim\left(\mathcal{C}^{(6)}\right)^{2} \frac{v^{4}}{\Lambda^{4}}$ would be inconsistent since they are in principle larger than the SM one-loop contributions, we have to drop the terms $\sim\left(\mathcal{C}^{(6)}\right)^{2} \frac{v^{4}}{\Lambda^{4}} \frac{g_{\mathrm{SM}}^{2}}{16 \pi^{2}}$ that are of the same order of the contributions from dimension- 8 operators. From a practical point of view, however, those terms are for the most part numerically irrelevant, and simply squaring the amplitude (22) after dropping the terms of order $\frac{v^{4}}{\Lambda^{4}}$ is a valid option. Lastly, we notice that the condition $\mathcal{C}^{(6)} \frac{v^{2}}{\Lambda^{2}}>\frac{g_{\mathrm{SM}}^{3}}{16 \pi^{2}}$ ensures that the fourth term in Eq. (22) is larger than a generic SM two-loop contribution.

\section{RESULTS}

$$
\text { A. } H \rightarrow Z Z
$$

In terms of the physical input parameters, $M_{W}, M_{Z}$, and $G_{\mu}$, the tree-level SMEFT amplitude for the on-shell decay $H \rightarrow Z^{\mu}\left(p_{1}\right) Z^{\nu}\left(p_{2}\right)$ is

$$
\begin{aligned}
\mathcal{A}^{0 l, \mu \nu}= & \mathcal{A}_{0, \mathrm{SM}}\left\{\left[T_{\mathrm{SM}}^{0 l}+\frac{1}{\Lambda^{2}} \Sigma_{i} \mathcal{C}_{i} T_{i}^{0 l}\right]\left(g^{\mu \nu}-\frac{p_{2}^{\mu} p_{1}^{\nu}}{p_{1} \cdot p_{2}}\right)\right. \\
& \left.+\left[T_{\mathrm{SM}}^{0 l}+\frac{1}{\Lambda^{2}} \Sigma_{i} \mathcal{C}_{i} \hat{T}_{i}^{0 l}\right]\left(\frac{p_{2}^{\mu} p_{1}^{\nu}}{p_{1} \cdot p_{2}}\right)\right\},
\end{aligned}
$$

where $\mathcal{A}_{0, \mathrm{SM}}=2^{5 / 4} \sqrt{G_{\mu}} M_{Z}^{2}, T_{\mathrm{SM}}^{0 l}=1$, the sum is over all of the contributing Warsaw basis coefficients $\mathcal{C}_{i}$, and the tree-level SMEFT contribution is

$$
\begin{aligned}
\Sigma_{i} T_{i}^{0 l} \mathcal{C}_{i} & =\frac{1}{\sqrt{2} G_{\mu}}\left(\frac{c_{k}}{2}\right)-\frac{2 p_{1} \cdot p_{2}}{\sqrt{2} G_{\mu} M_{Z}^{2}} c_{Z Z}, \\
\Sigma_{i} \hat{T}_{i}^{0 l} \mathcal{C}_{i} & =\frac{2}{\sqrt{2} G_{\mu} M_{Z}^{2}} c_{Z Z} .
\end{aligned}
$$

Note that the tree-level amplitude depends on only two combinations of coefficients,

$$
\begin{aligned}
c_{k} & \equiv \frac{\mathcal{C}_{\phi D}}{2}+2 \mathcal{C}_{\phi \square}+\mathcal{C}_{\mu e e \mu}^{l l}-2 \mathcal{C}_{\phi l}^{(3)} 3, \\
c_{Z Z} & \equiv\left[\mathcal{C}_{\phi W} \frac{M_{W}^{2}}{M_{Z}^{2}}+\left(1-\frac{M_{W}^{2}}{M_{Z}^{2}}\right) \mathcal{C}_{\phi B}+\frac{M_{W}}{M_{Z}} \sqrt{1-\frac{M_{W}^{2}}{M_{Z}^{2}}} \mathcal{C}_{\phi W B}\right] .
\end{aligned}
$$

The combination $c_{Z Z}$ is limited from global fits to the SMEFT contributions to Higgs decays. The 95\% confidence-level limit is $[15]^{3}$

\footnotetext{
${ }^{3}$ Note the differing normalization of $c_{Z Z}$ from Ref. [15].
} 


$$
-1.2\left(\frac{1 \mathrm{TeV}}{\Lambda^{2}}\right)<c_{Z Z}<1.6\left(\frac{1 \mathrm{TeV}}{\Lambda^{2}}\right)
$$

The tree-level decay width in the SMEFT is

$$
\begin{aligned}
\Gamma(H & \rightarrow Z Z)_{\mathrm{EFT}}^{0 l} \\
= & \frac{\beta G_{\mu} M_{H}^{3}}{16 \pi \sqrt{2}}\left(12 x^{2}-4 x+1\right)\left\{1+\frac{1}{\sqrt{2} G_{\mu} \Lambda^{2}} c_{k}\right\} \\
& +\frac{3 \beta M_{H}}{4 \pi} \frac{M_{Z}^{2}}{\Lambda^{2}} c_{Z Z}(2 x-1) \\
= & \Gamma(H \rightarrow Z Z)_{\mathrm{SM}}\left\{1+\frac{1}{\sqrt{2} G_{\mu} \Lambda^{2}}\right. \\
& \left.\times\left[c_{k}+\frac{24 x(2 x-1)}{12 x^{2}-4 x+1} c_{Z Z}\right]\right\},
\end{aligned}
$$

where $\beta=\sqrt{1-\frac{4 M_{Z}^{2}}{M_{H}^{2}}}$ and $x \equiv M_{Z}^{2} / M_{H}^{2}$. For $M_{H}$ between $2 M_{Z}$ and $200 \mathrm{GeV}$, the dependence on $M_{H}$ is minimal [4],

$$
\begin{aligned}
R^{0 l} & \equiv \frac{\Gamma(H \rightarrow Z Z)_{\mathrm{EFT}}^{0 l}}{\Gamma(H \rightarrow Z Z)_{\mathrm{SM}}^{0 l}} \\
& \sim 1+\frac{1}{\sqrt{2} G_{\mu} \Lambda^{2}}\left[c_{k}-4 c_{Z Z}\right]+\mathcal{O}\left(\mathcal{C} \frac{{ }^{2}{ }^{4}}{\Lambda^{4}}\right)
\end{aligned}
$$

Using the results of Appendix A, we can compare the onshell tree-level result of Eq. (29) with the off-shell result appropriate for the physical Higgs mass [30-32],

$$
\begin{aligned}
R^{\mathrm{off}} & \equiv \frac{\Gamma(H \rightarrow Z f \bar{f})^{0 l}}{\Gamma(H \rightarrow Z f \bar{f})_{\mathrm{SM}}} \\
& \sim 1+\frac{1}{\sqrt{2} G_{\mu} \Lambda^{2}}\left[c_{k}-.97 c_{\mathrm{ZZ}}\right]+\mathcal{O}\left(\mathcal{C} \frac{{ }^{2} v^{4}}{\Lambda^{4}}\right)
\end{aligned}
$$

Comparing Eqs. (29) and (30), we note that the off-shell effects are large [33].

In the SMEFT, the dimension-6 NLO results contain both the complete SM one-loop electroweak corrections to $H \rightarrow Z Z$ and the one-loop corrections from the dimension6 SMEFT operators. The complete, renormalized NLO amplitude is

$$
\mathcal{A}_{\mathrm{NLO}}^{\mu \nu}=\mathcal{A}^{0 l, \mu \nu}+\mathcal{A}^{1 l, \mu \nu}
$$

The one-loop SMEFT contribution to the amplitude can be expanded as

$$
\begin{aligned}
\mathcal{A}^{1 l, \mu \nu}= & \mathcal{A}_{0, \mathrm{SM}}\left\{\left(T_{\mathrm{SM}}^{1 l}+\mathcal{F}_{g} \log \left(\frac{\Lambda^{2}}{M_{Z}^{2}}\right)\right.\right. \\
& \left.+\frac{(1 \mathrm{TeV})^{2}}{\Lambda^{2}} \sum_{i} T_{i}^{1 l} \mathcal{C}_{i}\right)\left(g^{\mu \nu}-\frac{p_{1}^{\nu} p_{2}^{\mu}}{p_{1} \cdot p_{2}}\right) \\
& +\left(\hat{T}_{\mathrm{SM}}^{1 l}+\mathcal{F}_{p} \log \left(\frac{\Lambda^{2}}{M_{Z}^{2}}\right)\right. \\
& \left.\left.+\frac{(1 \mathrm{TeV})^{2}}{\Lambda^{2}} \sum_{i} \hat{T}_{i}^{1 l} \mathcal{C}_{i}\right) \frac{p_{1}^{\nu} p_{2}^{\mu}}{p_{1} \cdot p_{2}}\right\}
\end{aligned}
$$

where the terms $\mathcal{F}_{g} \log \left(\frac{\Lambda^{2}}{M_{Z}^{2}}\right)$ and $\mathcal{F}_{p} \log \left(\frac{\Lambda^{2}}{M_{Z}^{2}}\right)$ contain the residual dependence on the renormalization scale, due to our choice of renormalization scheme. Retaining terms to $\mathcal{O}\left(\frac{v^{2}}{\Lambda^{2}}\right)$, we parametrize the exact one-loop SMEFT result, ${ }^{4}$

$$
\begin{aligned}
T_{i}^{l l}= & a_{0, i}+a_{1, i} \frac{M_{H}^{2}}{M_{Z}^{2}}+a_{2, i}\left(\frac{M_{H}^{2}}{M_{Z}^{2}}\right)^{2}+a_{3, i} \log \left(\frac{M_{H}^{2}}{M_{Z}^{2}}\right) \\
& +a_{4, i} \log \left(\frac{4 M_{t}^{2}-M_{H}^{2}}{M_{Z}^{2}}\right) \\
\hat{T}_{i}^{1 l}= & b_{0, i}+b_{1, i} \frac{M_{H}^{2}}{M_{Z}^{2}}+b_{2, i}\left(\frac{M_{H}^{2}}{M_{Z}^{2}}\right)^{2}+b_{3, i} \log \left(\frac{M_{H}^{2}}{M_{Z}^{2}}\right) \\
& +a_{4, i} \log \left(\frac{4 M_{t}^{2}-M_{H}^{2}}{M_{Z}^{2}}\right) .
\end{aligned}
$$

Numerical values for the fit parameters, together with the analytical expressions for $\mathcal{F}_{g}$ and $\mathcal{F}_{p}$ are given in Appendix B.

The SM result is easily recovered,

$\mathcal{A}_{\mathrm{SM}}^{1 l, \mu \nu}=\mathcal{A}_{0, \mathrm{SM}}\left\{T_{\mathrm{SM}}^{1 l}\left(g^{\mu \nu}-\frac{p_{1}^{\nu} p_{2}^{\mu}}{p_{1} \cdot p_{2}}\right)+\hat{T}_{\mathrm{SM}}^{1 l} \frac{p_{1}^{\nu} p_{2}^{\mu}}{p_{1} \cdot p_{2}}\right\}$,

where $T_{\mathrm{SM}}^{1 l}=a_{0, \mathrm{SM}}$ and $\hat{T}_{\mathrm{SM}}^{1 l}=b_{0, \mathrm{SM}}$. We have verified that our one-loop electroweak SM corrections are in agreement with previous results [18].

In Fig. 1, we illustrate the dependence of the decay widths on the terms retained in the expansion of Eq. (23). The curves labeled " $1 / \Lambda^{2}$ expansion" drop the $\hat{a}_{1}^{2}$ and $\left(\hat{a}_{0} \hat{a}_{4}+\hat{a}_{1} \hat{a}_{3}\right)$ contributions in Eq. (23). It is apparent that for large values of the $\mathcal{C}$ (here $\mathcal{C}_{\phi W}$ ) the expansion is nonsense. For $\mathcal{C}_{\phi W}>2$, the dimension-6 approximation breaks down, and the dimension- 6 approximation to the amplitude squared becomes negative. The curves labeled " $|\mathcal{A}|^{2}$ " contain all of the terms in the square of Eq. (22) (with $\hat{a}_{4} \rightarrow 0$ ) and are well behaved for all $\mathcal{C}$. For $\mathcal{C}_{\phi W}<2$, the full amplitude squared is well approximated by the terms linear in the coefficient functions, and the SMEFT

\footnotetext{
${ }^{4}$ This fit is valid for $M_{H}<2 M_{t}$.
} 


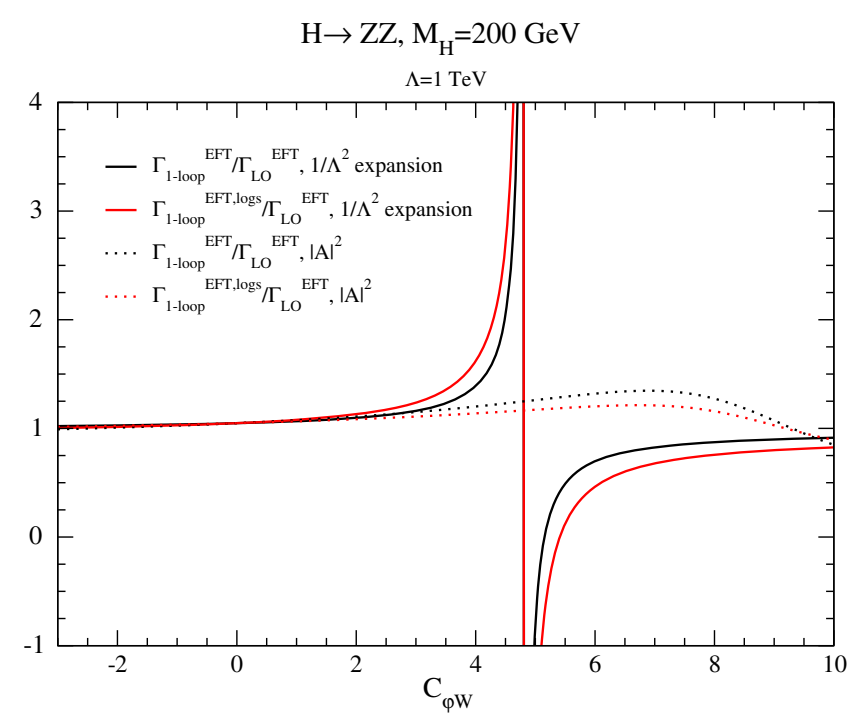

FIG. 1. Dependence of $H \rightarrow Z Z$ decay width on $\mathcal{C}_{\phi W}$ in different expansions as explained in the text.

dimension-6 approximation is valid. We have checked that this condition is satisfied for the following plots in this section.

In Fig. 2, we show the contribution relative to the LO SM prediction for representative values of the SMEFT coefficients that contribute at tree level. Choosing all coefficients positive, for the parameters we have chosen, inclusion of the tree-level SMEFT coefficients decreases the rate by $\sim 2 \%$ (red curve). The SM one-loop corrections (black curve) increase the rate by roughly $5 \%$, leading to a partial cancellation between the one-loop SM and tree-level SMEFT contributions. This makes it clear that global fits to SMEFT coefficients that do not contain the electroweak corrections cannot be more accurate than $\sim \mathcal{O}(5 \%)$. The one-loop corrections from the SMEFT operators are much smaller than the SM electroweak corrections. In this example, the complete NLO SMEFT calculation is well approximated by including only the logarithmic contributions from the SMEFT coefficients. On the rhs of Fig. 2, we flip the sign of the SMEFT coefficients, which increases their numerical significance. Note that the dependence on $M_{H}$ is rather mild.

In Figs. 3 and 5, we show the dependence on SMEFT coefficients as a function of the $\mathcal{C}$ for fixed $M_{H}=200 \mathrm{GeV}$, normalized to the LO SMEFT result. Figure 3 shows the dependence on coefficients that enter at tree level, and in Fig. 4, we replot the same results, normalized to the one-loop SM. The change from the one-loop SM results in the SMEFT is of $\mathcal{O}(5 \%)$ for $\mathcal{C}_{\phi B} \sim \mathcal{O}(-1)$, and for $\mathcal{C}_{\phi W} \sim$ $\mathcal{O}(-1)$ this change is $\mathcal{O}(20 \%)$. These values are consistent with current fits to LHC Higgs decays to ZZ.

Figure 5 shows the dependence on a selection of coefficient functions that do not enter at tree level. An interesting feature of our results is that they can be used to obtain limits on coefficients that first enter at loop level. For example, from a global fit $[15,16,34]$,

$$
\begin{aligned}
\mathcal{C}_{W} & =\frac{\Lambda^{2}}{v^{2}}(1.14 \pm .68) \\
& \rightarrow(18.8 \pm 11.2)\left(\frac{\Lambda^{2}}{1 \mathrm{TeV}^{2}}\right) .
\end{aligned}
$$

Such a large value of $\mathcal{C}_{W}$ would increase the LO SMEFT width to $Z Z$ by $\sim 20 \%$ as observed in Fig. 5. Loop corrections to Higgs decays therefore have the possibility of new constraints on the SMEFT coefficients.

Our corrections to the on-shell $H \rightarrow Z Z$ one-loop SMEFT result must be considered as a first step in a full
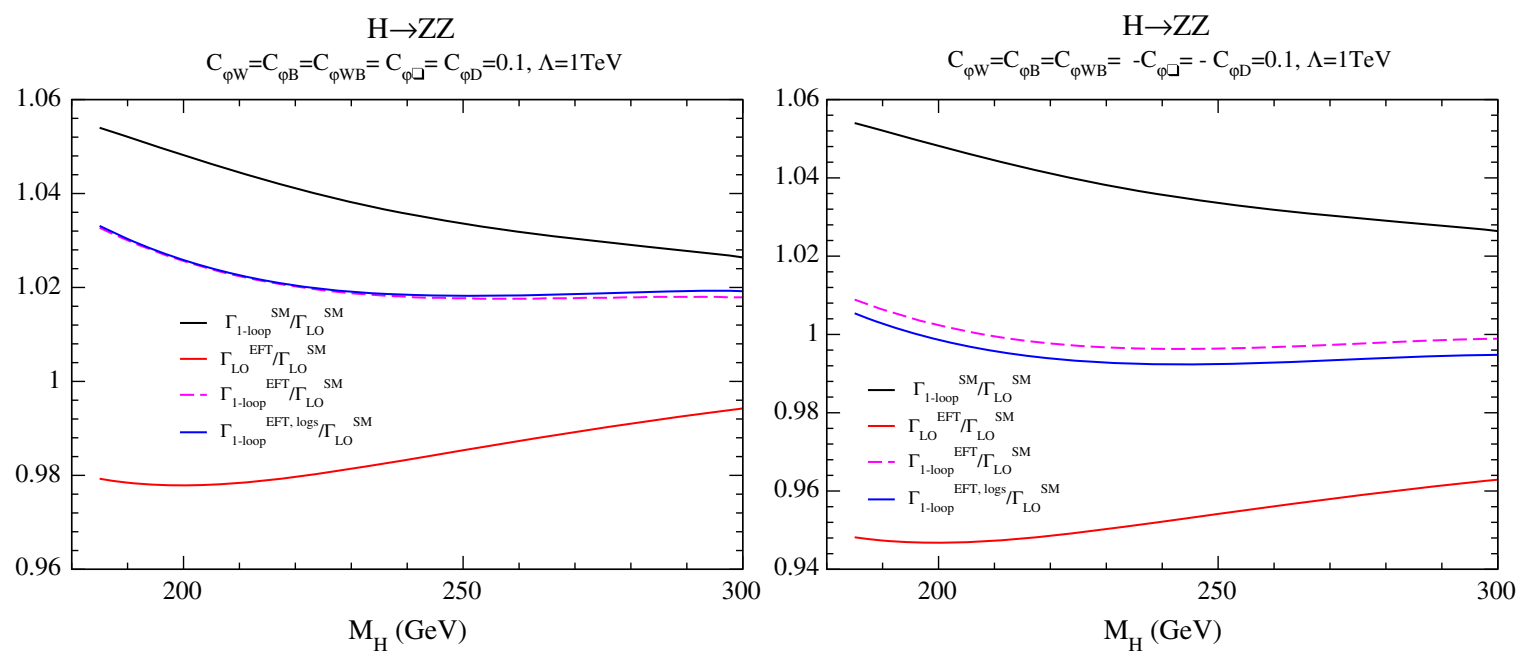

FIG. 2. Dependence of the $H \rightarrow Z Z$ decay width on SMEFT coefficients that contribute at tree level. Note that the curve labeled "oneloop EFT" is the complete SMEFT result and contains both the one-loop SM result and the one-loop contribution from the dimension-6 SMEFT coefficients. Coefficients not specified are set to 0 . 

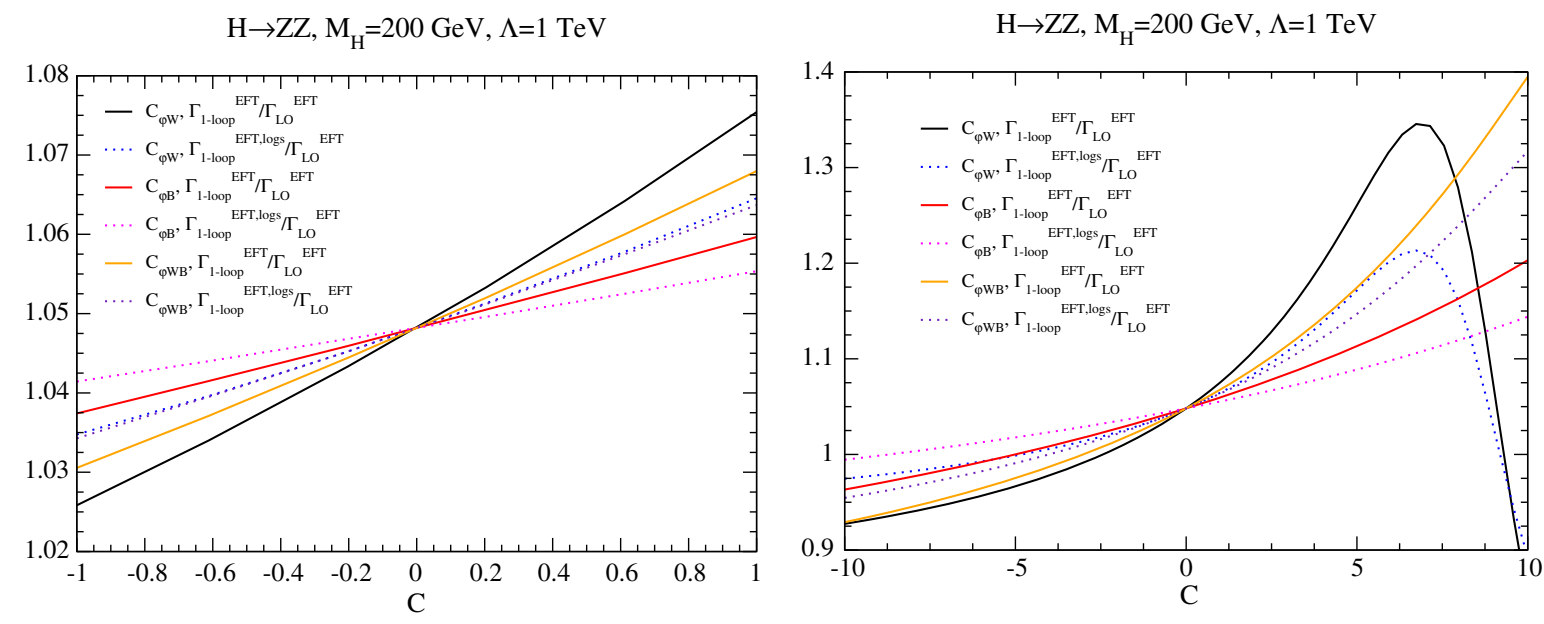

FIG. 3. Dependence of $H \rightarrow Z Z$ decay width on SMEFT coefficients. Coefficients are varied one at a time, and coefficients not specified are set to 0 .

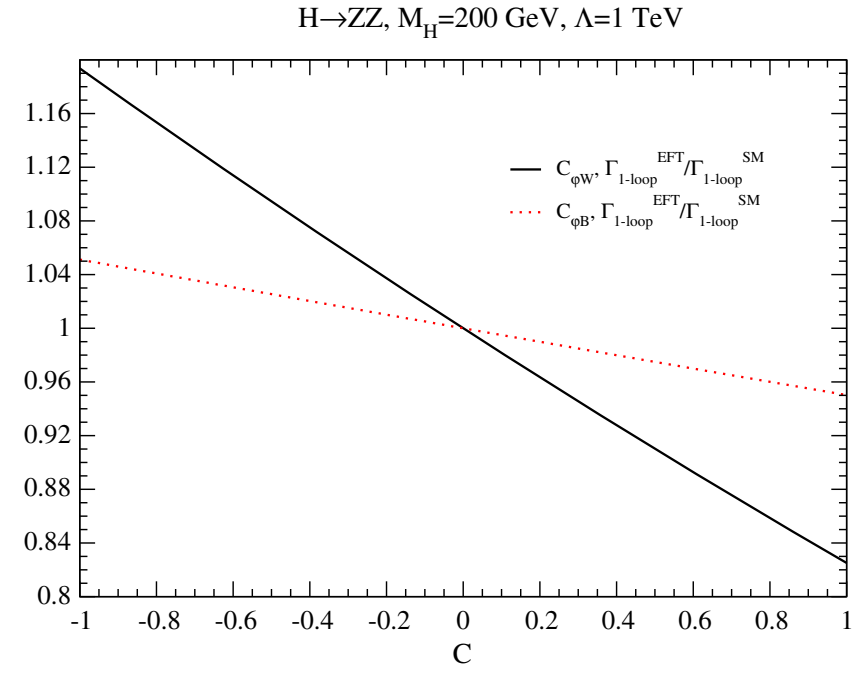

FIG. 4. Same as Fig. 3, but normalized to $\Gamma_{1-\text { loop }}^{\mathrm{SM}}$.
SMEFT calculation for $H \rightarrow Z f \bar{f}$. Our results suggest, however, that the results of Figs. 3 and 5 can be thought of as $K$ factors that multiply the LO SMEFT off-shell result of Eq. (30). The numerical size of our results implies that a full SMEFT calculation is needed for $H \rightarrow Z f \bar{f}$ in order to obtain reliable results. The SM electroweak corrections for $H \rightarrow Z f \bar{f}$ are known and can be implemented using the PROPHECY4F program $[35,36]$.

\section{B. $\boldsymbol{H} \rightarrow \mathrm{Z} \gamma$}

The one-loop SMEFT results for $H \rightarrow Z^{\mu}\left(p_{1}\right) \gamma^{\nu}\left(p_{2}\right)$ can be obtained in a straightforward manner from the results of the previous section. At tree level, there is an SMEFT contribution, while the SM contribution begins at one loop. The Ward identity for the photon requires $p^{\nu} \cdot \mathcal{A}_{\mu \nu}=0$, so the tensor structure is fixed,

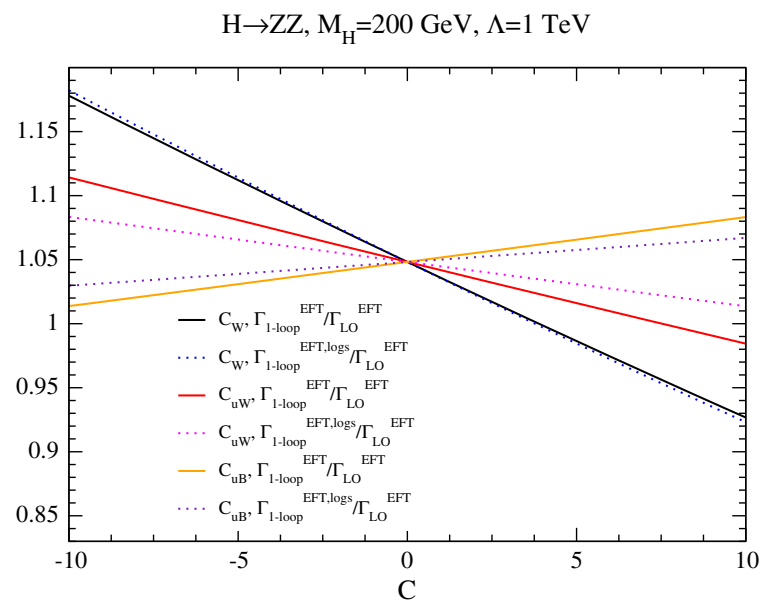

FIG. 5. Dependence of the one-loop corrected SMEFT width on various $\mathcal{C}$ for $M_{H}=200 \mathrm{GeV}$. 
$\mathcal{A}_{Z \gamma}^{\mu \nu}=\left[\mathcal{A}_{Z \gamma, \mathrm{EFT}}^{0 l}+\mathcal{A}_{Z \gamma, \mathrm{SM}}^{1 l}+\mathcal{A}_{Z \gamma, \mathrm{EFT}}^{1 l}\right]\left(g_{\mu \nu}-\frac{p_{1}^{\nu} p_{2}^{\mu}}{p_{1} \cdot p_{2}}\right)$.

The tree-level SMEFT contribution is

$$
\mathcal{A}_{Z \gamma, \mathrm{EFT}}^{0 l}=-2^{3 / 4} G_{\mu}^{-1 / 2}\left(\frac{M_{H}^{2}-M_{Z}^{2}}{\Lambda^{2}}\right) c_{Z \gamma}
$$

with

$$
c_{Z \gamma} \equiv \frac{M_{W}}{M_{Z}} \sqrt{1-\frac{M_{W}^{2}}{M_{Z}^{2}}}\left(\mathcal{C}_{\phi W}-\mathcal{C}_{\phi B}\right)+\left(1-2 \frac{M_{W}^{2}}{M_{Z}^{2}}\right) \mathcal{C}_{\phi W B} .
$$

Numerically, for $M_{H}=125 \mathrm{GeV}$ and $\Lambda=1 \mathrm{TeV}$,

$$
\mathcal{A}_{Z \gamma, \mathrm{EFT}}^{0 l}=1.5 \mathrm{GeV}\left(\mathcal{C}_{\phi W}-\mathcal{C}_{\phi B}-1.3 \mathcal{C}_{\phi W B}\right)\left(\frac{1 \mathrm{TeV}}{\Lambda^{2}}\right)
$$

The SM contribution is well known,

$$
\begin{aligned}
\mathcal{A}_{Z \gamma, \mathrm{SM}}^{1 l}= & \left(\frac{M_{H}^{2}-M_{Z}^{2}}{2}\right) \frac{M_{W}^{2}}{\pi^{2}} G_{\mu}^{3 / 2} 2^{-1 / 4} \sqrt{1-\frac{M_{W}^{2}}{M_{Z}^{2}}} \\
& \times\left\{\Sigma_{f} N_{c} \frac{Q_{f} M_{Z}}{M_{W}} v_{f} A_{1 / 2}\left(\tau_{f}, \lambda_{f}\right)+A_{1}\left(\tau_{W}, \lambda_{W}\right)\right\} \\
= & 0.209 \mathrm{GeV} \quad \text { for } M_{H}=125 \mathrm{GeV},
\end{aligned}
$$

where the sum is over all fermions, $N_{c}=1(3)$ for leptons (quarks), $\quad \tau_{i}=4 M_{i}^{2} / M_{H}^{2}, \quad \lambda_{i}=4 M_{i}^{2} / M_{Z}^{2}, \quad v_{f}=2 T_{f}^{3}-$ $4 Q_{f}\left(1-\frac{M_{W}^{2}}{M_{Z}^{2}}\right)$, and analytic expressions for the functions $A_{1}$ and $A_{1 / 2}$ can be found in Refs. [19,20,37].

We report our one-loop SMEFT corrections to the $H \rightarrow Z \gamma$ amplitude, $\mathcal{A}_{Z \gamma}^{1 l}$, numerically for $M_{H}=125 \mathrm{GeV}$,

$$
\begin{aligned}
\mathcal{A}_{Z \gamma, \mathrm{EFT}}^{l l}= & \frac{(1 \mathrm{TeV})^{2}}{\Lambda^{2}}\left\{-0.038 \mathcal{C}_{\phi l}^{(3)}+0.00185\left(\mathcal{C}_{\phi q}^{(1)}-\mathcal{C}_{\phi q}^{(3)}+\mathcal{C}_{\phi u}\right)-0.0126 \mathcal{C}_{\phi D}\right. \\
& +0.0127 \mathcal{C}_{\phi \square}+0.000753 \mathcal{C}_{u \phi}+0.019 \mathcal{C}_{l l}+\left(0.00778-0.0362 \log \frac{\Lambda^{2}}{M_{Z}^{2}}\right) \mathcal{C}_{\phi B} \\
& +\left(-0.00158+0.0154 \log \frac{\Lambda^{2}}{M_{Z}^{2}}\right) \mathcal{C}_{\phi W}+\left(-0.0524-0.0269 \log \frac{\Lambda^{2}}{M_{Z}^{2}}\right) \mathcal{C}_{\phi W B} \\
& +\left(-0.00999+0.0042 \log \frac{\Lambda^{2}}{M_{Z}^{2}}\right) \mathcal{C}_{u B}+\left(0.0669-0.0295 \log \frac{\Lambda^{2}}{M_{Z}^{2}}\right) \mathcal{C}_{u W} \\
& \left.+\left(0.00559-0.0213 \log \frac{\Lambda^{2}}{M_{Z}^{2}}\right) \mathcal{C}_{W}\right\}
\end{aligned}
$$

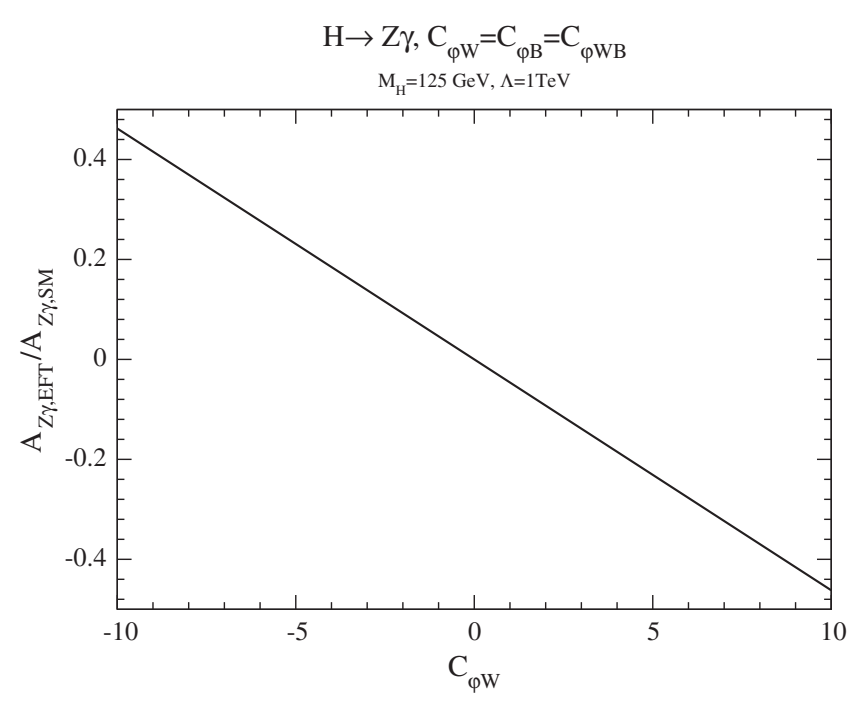

FIG. 6. Contribution to $H \rightarrow Z \gamma$ in the SMEFT. The vertical axis is the ratio of the one-loop contribution of Eq. (41) to the SM one-loop result of Eq. (40).
[Note that Eq. (41) is in GeV.] An interesting feature of Eq. (41) is the dependence on coefficients not arising at tree level.

The contribution from the SMEFT operators to $H \rightarrow Z \gamma$ at one loop is much smaller than the SM one-loop contribution, due to the $\frac{v^{2}}{\Lambda^{2}}$ suppression. The contribution to the amplitude, Eq. (41), is shown in Fig. 6.

\section{CONCLUSIONS}

We have computed the one-loop electroweak corrections in the SMEFT to the on-shell $H \rightarrow Z Z$ process. Numerically, the logarithmic SMEFT contributions dominate over the finite NLO contributions for most of parameter space. Appendix B contains a numerical fit to the finite NLO SMEFT contributions, and Appendix $\mathrm{C}$ has analytic results for the logarithmic contributions to $H \rightarrow Z Z$. Our complete result can be obtained from the ancillary files provided as Supplemental Material [38]. The calculation of the on-shell decay is a first step toward 
a full NLO calculation of the physical $H \rightarrow Z f \bar{f}$ process, and our results can be used to approximate a $K$ factor for the SMEFT $H \rightarrow Z f \bar{f}$ decay.

The full $H \rightarrow Z \gamma$ SMEFT NLO result is presented as a byproduct of our calculation. Finally, the complete result for the one-loop SMEFT renormalization of $G_{\mu}$ is given for the first time.

\section{ACKNOWLEDGMENTS}

We thank Giuseppe Degrassi for discussions. S. D. and P. P. G. are supported by the U.S. Department of Energy under Grant No. de-sc0012704.

\section{APPENDIX A: OFF-SHELL PRODUCTION}

The decay width for the off-shell decay, $H \rightarrow$ $f_{1}\left(p_{1}\right) f_{2}\left(p_{2}\right) Z\left(p_{3}\right)$, is

$$
\Gamma=\int_{0}^{\left(M_{H}-M_{Z}\right)^{2}} d q^{2} \int d m_{23}^{2} \frac{|A|^{2}}{256 \pi^{3} M_{H}^{3}}
$$

where $m_{i j}=\left(p_{i}+p_{j}\right)^{2}, m_{12}^{2} \equiv q^{2}, m_{12}^{2}+m_{23}^{2}+m_{13}^{2}=$ $M_{H}^{2}+M_{Z}^{2}, \quad \lambda\left(M_{H}^{2}, M_{Z}^{2}, q^{2}\right) \equiv q^{4}-2 q^{2}\left(M_{H}^{2}+M_{Z}^{2}\right)+\left(M_{H}^{2}-\right.$ $\left.M_{Z}^{2}\right)^{2}$, and $\left.m_{23}^{2}\right|_{\max , \min } \equiv \frac{1}{2}\left(M_{H}^{2}+M_{Z}^{2}-q^{2} \pm \sqrt{\lambda}\right)$. We write the amplitude squared to $\mathcal{O}\left(\frac{1}{\Lambda^{2}}\right)$ in the SMEFT as

$$
\left|A_{\mathrm{EFT}}\right|^{2}=\left|A_{\mathrm{SM}}\right|^{2}+\left|\delta A_{\mathrm{EFT}}\right|^{2}+\mathcal{O}\left(\mathcal{C}^{2} \frac{v^{4}}{\Lambda^{4}}\right)
$$

where

$$
\begin{aligned}
\left|A_{\mathrm{SM}}\right|^{2} & =32\left(g_{L}^{2}+g_{R}^{2}\right) G_{F}^{2} M_{Z}^{4}\left[\frac{2 M_{Z}^{2} q^{2}-m_{13}^{2} q^{2}-M_{H}^{2} M_{Z}^{2}+m_{13}^{2} M_{Z}^{2}+m_{13}^{2} M_{H}^{2}-m_{13}^{4}}{\left(q^{2}-M_{Z}^{2}\right)^{2}+\Gamma_{Z}^{2} M_{Z}^{2}}\right] \\
\left|\delta A_{\mathrm{EFT}}\right|^{2} & =\left|A_{\mathrm{SM}}\right|^{2} \frac{1}{\sqrt{2} G_{F} \Lambda^{2}} c_{k}+64\left(g_{L}^{2}+g_{R}^{2}\right) \frac{\sqrt{2} G_{F}}{\Lambda^{2}} M_{Z}^{4} \frac{q^{2}\left(q^{2}+M_{Z}^{2}-M_{H}^{2}\right)}{\left(q^{2}-M_{Z}^{2}\right)^{2}+\Gamma_{Z}^{2} M_{Z}^{2}} c_{Z Z} .
\end{aligned}
$$

Note that we have not included effects due to possible anomalous $H \rightarrow Z \gamma$ vertices here, although they are included in the results of Sec. IV B.

Integrating over $d m_{23}^{2}$,

$$
\begin{aligned}
\left.\frac{d \Gamma}{d q^{2}}\right|_{\mathrm{SM}}= & \left(g_{L}^{2}+g_{R}^{2}\right) G_{F}^{2} \sqrt{\lambda\left(M_{H}^{2}, M_{Z}^{2}, q^{2}\right)} \frac{M_{Z}^{4}}{48 \pi^{3} M_{H}^{3}}\left[\frac{\left(12 M_{Z}^{2} q^{2}+\lambda\left(M_{H}^{2}, M_{Z}^{2}, q^{2}\right)\right)}{\left(q^{2}-M_{Z}^{2}\right)^{2}+\Gamma_{Z}^{2} M_{Z}^{2}}\right] \\
\left.\frac{d \Gamma}{d q^{2}}\right|_{\mathrm{EFT}}= & \left.\frac{d \Gamma}{d q^{2}}\right|_{\mathrm{SM}}\left\{1+\frac{1}{\sqrt{2} G_{F} \Lambda^{2}} c_{k}\right\}+\left(g_{L}^{2}+g_{R}^{2}\right) \frac{G_{F}}{\Lambda^{2}} \sqrt{\lambda\left(M_{H}^{2}, M_{Z}^{2}, q^{2}\right)} \frac{M_{Z}^{4}}{2 \sqrt{2} \pi^{3} M_{H}^{3}} \\
& \cdot \frac{q^{2}\left(3 M_{Z}^{2}+M_{H}^{2}\right)-\left(M_{Z}^{2}-M_{H}^{2}\right)^{2}+\lambda\left(M_{H}^{2}, M_{Z}^{2}, q^{2}\right)}{\left(q^{2}-M_{Z}^{2}\right)^{2}+\Gamma_{Z}^{2} M_{Z}^{2}} c_{Z Z} .
\end{aligned}
$$

Finally, integrating over $q^{2}$ and setting $M_{H}=125 \mathrm{GeV}$,

$$
\begin{aligned}
R^{\mathrm{off}} & \equiv \frac{\int d q^{2} d \Gamma /\left.d q^{2}\right|_{\mathrm{EFT}}}{\int d q^{2} d \Gamma / d q_{\mathrm{SM}}^{2}} \\
& \sim 1+\frac{1}{\sqrt{2} G_{F} \Lambda^{2}}\left\{c_{k}-.97 c_{\mathrm{ZZ}}\right\} .
\end{aligned}
$$

This is in agreement with Refs. [31,39,40]. Note that if we require a minimum $q^{2} \equiv q_{\text {cut }}^{2}$ the result is altered. Define

$$
\begin{aligned}
R^{\text {off }}\left(q_{\text {cut }}^{2}\right) & \equiv \frac{\int_{q_{\text {cut }}^{2}}^{\left(M_{H}-M_{Z}\right)^{2}} d q^{2} d \Gamma /\left.d q^{2}\right|_{\mathrm{EFT}}}{\int_{q_{\text {cut }}^{2}}^{\left(M_{H}-M_{Z}\right)^{2}} d q^{2} d \Gamma / d q_{\mathrm{SM}}^{2}} \\
& =1+\frac{1}{\sqrt{2} G_{F} \Lambda^{2}}\left\{c_{k}+f\left(q_{2}^{\mathrm{cut}}\right) c_{Z Z}\right\} .
\end{aligned}
$$

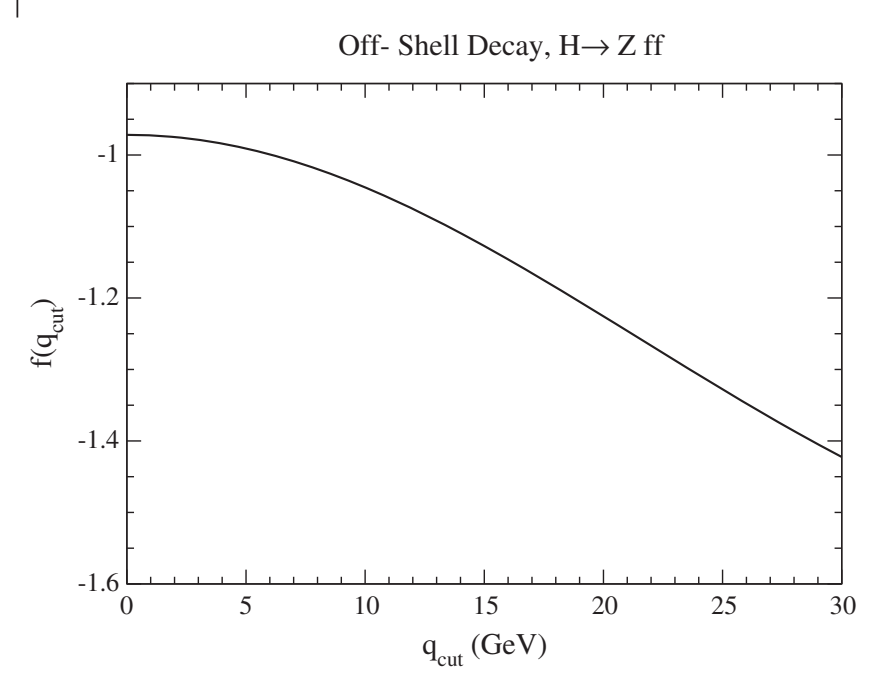

FIG. 7. Dependence of the off-shell decay rate on the $q_{\text {cut }}$ defined in Eq. (A6). 
The effects of the $q_{\text {cut }}^{2}$ are shown in Fig. 7. References [30,33] note the numerically large effect of off-shell Z's, which is seen clearly in the sensitivity to the $q_{\text {cut }}^{2}$.

\section{APPENDIX B: NUMERICAL VALUES FOR THE ON-SHELL DECAY $H \rightarrow Z Z$}

Numerical values for the one-loop SMEFT on-shell decay $H \rightarrow Z Z$ in terms of the parametrization of Eq. (33) are given in Tables II-III,

TABLE II. Numerical values for the coefficients defined in Eq. (33) relevant for the on-shell process $H \rightarrow Z Z$.

\begin{tabular}{lccccc}
\hline \hline & $a_{0}$ & $a_{1}$ & $a_{2}$ & $a_{3}$ & $a_{4}$ \\
\hline $\mathrm{SM}$ & $-3.09 \times 10^{-2}$ & $-1.41 \times 10^{-2}$ & $5.43 \times 10^{-4}$ & $4.93 \times 10^{-2}$ & $1.57 \times 10^{-2}$ \\
$\mathcal{C}_{W}$ & $-3.08 \times 10^{-3}$ & $-8.96 \times 10^{-5}$ & $4.76 \times 10^{-5}$ & $1.43 \times 10^{-4}$ & $8.29 \times 10^{-4}$ \\
$\mathcal{C}_{\phi}$ & $-2.12 \times 10^{-3}$ & $-2.11 \times 10^{-4}$ & $6.59 \times 10^{-6}$ & $1.41 \times 10^{-3}$ & $1.2 \times 10^{-4}$ \\
$\mathcal{C}_{\phi \square}$ & $2.99 \times 10^{-3}$ & $2.11 \times 10^{-4}$ & $2.9 \times 10^{-5}$ & $-1.14 \times 10^{-4}$ & $1.23 \times 10^{-3}$ \\
$\mathcal{C}_{\phi D}$ & $1.42 \times 10^{-3}$ & $-1.63 \times 10^{-5}$ & $7.09 \times 10^{-6}$ & $4.86 \times 10^{-4}$ & $1.51 \times 10^{-4}$ \\
$\mathcal{C}_{u \phi}$ & $1.56 \times 10^{-3}$ & $-6.49 \times 10^{-6}$ & $1.3 \times 10^{-6}$ & $3.29 \times 10^{-5}$ & $-4.65 \times 10^{-4}$ \\
$\mathcal{C}_{\phi W}$ & $1.14 \times 10^{-2}$ & $8.69 \times 10^{-4}$ & $-1.75 \times 10^{-4}$ & $-1.02 \times 10^{-2}$ & $-7.56 \times 10^{-4}$ \\
$\mathcal{C}_{\phi B}$ & $3.52 \times 10^{-3}$ & $2.64 \times 10^{-5}$ & $-8.25 \times 10^{-5}$ & $-2.02 \times 10^{-3}$ & $-5.46 \times 10^{-4}$ \\
$\mathcal{C}_{\phi W B}$ & $5.88 \times 10^{-3}$ & $-1.63 \times 10^{-3}$ & $-5.93 \times 10^{-5}$ & $1.62 \times 10^{-3}$ & $-8.97 \times 10^{-4}$ \\
$\mathcal{C}_{u W}$ & $-1.72 \times 10^{-3}$ & $7.28 \times 10^{-4}$ & $-4.99 \times 10^{-6}$ & $-5.86 \times 10^{-5}$ & $1.36 \times 10^{-4}$ \\
$\mathcal{C}_{u B}$ & $9.2 \times 10^{-4}$ & $-3.9 \times 10^{-4}$ & $2.67 \times 10^{-6}$ & $3.14 \times 10^{-5}$ & $-7.3 \times 10^{-5}$ \\
$\mathcal{C}_{\phi l}^{(1)}$ & $6.82 \times 10^{-7}$ & 0 & 0 & 0 & 0 \\
$\mathcal{C}_{\phi l}^{(3)}$ & $3.56 \times 10^{-3}$ & $2.56 \times 10^{-3}$ & $-9.87 \times 10^{-5}$ & $-8.97 \times 10^{-3}$ & $-2.85 \times 10^{-3}$ \\
$\mathcal{C}_{\phi e}$ & $6.26 \times 10^{-5}$ & 0 & 0 & 0 & 0 \\
$\mathcal{C}_{\phi q}^{(1)}$ & $4.45 \times 10^{-3}$ & $-2.23 \times 10^{-5}$ & $-9.49 \times 10^{-7}$ & $-1.64 \times 10^{-5}$ & $5.43 \times 10^{-5}$ \\
$\mathcal{C}_{\phi q}^{(3)}$ & $-6.06 \times 10^{-3}$ & $2.23 \times 10^{-5}$ & $9.49 \times 10^{-7}$ & $1.64 \times 10^{-5}$ & $-5.43 \times 10^{-5}$ \\
$\mathcal{C}_{\phi u}$ & $-4.71 \times 10^{-3}$ & $5.2 \times 10^{-5}$ & $2.14 \times 10^{-6}$ & $3.67 \times 10^{-5}$ & $-1.2 \times 10^{-4}$ \\
$\mathcal{C}_{\phi d}$ & $6.26 \times 10^{-5}$ & 0 & 0 & 0 & 0 \\
$\mathcal{C}_{l l}$ & $-3.17 \times 10^{-3}$ & $-1.28 \times 10^{-3}$ & $4.93 \times 10^{-5}$ & $4.49 \times 10^{-3}$ & $1.43 \times 10^{-3}$ \\
$\mathcal{C}_{l q}^{(3)}$ & $8.9 \times 10^{-4}$ & 0 & 0 & 0 & 0 \\
\hline \hline
\end{tabular}

TABLE III. Numerical values for the coefficients defined in Eq. (33) relevant for the on-shell process $H \rightarrow Z Z$.

\begin{tabular}{lccccc}
\hline \hline & $b_{0}$ & $b_{1}$ & $b_{2}$ & $b_{3}$ & $b_{4}$ \\
\hline $\mathrm{SM}$ & $-2.68 \times 10^{-2}$ & $-6.16 \times 10^{-3}$ & $2.83 \times 10^{-4}$ & $2.49 \times 10^{-2}$ & $1.03 \times 10^{-2}$ \\
$\mathcal{C}_{W}$ & $-1.6 \times 10^{-3}$ & $-4.44 \times 10^{-4}$ & $1.62 \times 10^{-5}$ & $1.76 \times 10^{-3}$ & $3.44 \times 10^{-4}$ \\
$\mathcal{C}_{\phi}$ & $-2.04 \times 10^{-3}$ & $-1.88 \times 10^{-4}$ & $5.84 \times 10^{-6}$ & $1.26 \times 10^{-3}$ & $1.06 \times 10^{-4}$ \\
$\mathcal{C}_{\phi \square}$ & $3.2 \times 10^{-3}$ & $7.73 \times 10^{-4}$ & $1.15 \times 10^{-5}$ & $-1.74 \times 10^{-3}$ & $8.63 \times 10^{-4}$ \\
$\mathcal{C}_{\phi D}$ & $1.51 \times 10^{-3}$ & $1.23 \times 10^{-4}$ & $3.13 \times 10^{-6}$ & $8.87 \times 10^{-5}$ & $4.19 \times 10^{-5}$ \\
$\mathcal{C}_{u \phi}$ & $1.52 \times 10^{-3}$ & $-7.6 \times 10^{-6}$ & $1.08 \times 10^{-6}$ & $2.86 \times 10^{-5}$ & $-4.49 \times 10^{-4}$ \\
$\mathcal{C}_{\phi W}$ & $6.37 \times 10^{-3}$ & $1.07 \times 10^{-3}$ & $-3.92 \times 10^{-5}$ & $-5.3 \times 10^{-3}$ & $-8.58 \times 10^{-4}$ \\
$\mathcal{C}_{\phi B}$ & $7.33 \times 10^{-4}$ & $9.47 \times 10^{-5}$ & $-3.39 \times 10^{-6}$ & $-4.85 \times 10^{-4}$ & $-7.1 \times 10^{-5}$ \\
\hline & & & & & (Table continued)
\end{tabular}


TABLE III. (Continued)

\begin{tabular}{lccccc}
\hline \hline & $b_{0}$ & $b_{1}$ & $b_{2}$ & $b_{3}$ & $b_{4}$ \\
\hline $\mathcal{C}_{\phi W B}$ & $8.71 \times 10^{-4}$ & $-1.19 \times 10^{-4}$ & $6.47 \times 10^{-6}$ & $1.17 \times 10^{-5}$ & $1.92 \times 10^{-4}$ \\
$\mathcal{C}_{u W}$ & $1.09 \times 10^{-5}$ & $-4.3 \times 10^{-8}$ & $-1.47 \times 10^{-8}$ & $-3.18 \times 10^{-7}$ & $1.48 \times 10^{-6}$ \\
$\mathcal{C}_{u B}$ & $-5.85 \times 10^{-6}$ & $2.3 \times 10^{-8}$ & $7.87 \times 10^{-9}$ & $1.7 \times 10^{-7}$ & $-7.93 \times 10^{-7}$ \\
$\mathcal{C}_{\phi l}^{(1)}$ & $6.82 \times 10^{-7}$ & 0 & 0 & 0 & 0 \\
$\mathcal{C}_{\phi l}^{(3)}$ & $2.8 \times 10^{-3}$ & $1.12 \times 10^{-3}$ & $-5.14 \times 10^{-5}$ & $-4.52 \times 10^{-3}$ & $-1.88 \times 10^{-3}$ \\
$\mathcal{C}_{\phi e}$ & $6.26 \times 10^{-5}$ & 0 & 0 & 0 & 0 \\
$\mathcal{C}_{\phi q}^{(1)}$ & $4.36 \times 10^{-3}$ & $-3.33 \times 10^{-5}$ & $-1.75 \times 10^{-6}$ & $-3.11 \times 10^{-5}$ & $1.06 \times 10^{-4}$ \\
$\mathcal{C}_{\phi q}^{(3)}$ & $-5.97 \times 10^{-3}$ & $3.33 \times 10^{-5}$ & $1.75 \times 10^{-6}$ & $3.11 \times 10^{-5}$ & $-1.06 \times 10^{-4}$ \\
$\mathcal{C}_{\phi u}$ & $-4.71 \times 10^{-3}$ & $3.33 \times 10^{-5}$ & $1.75 \times 10^{-6}$ & $3.11 \times 10^{-5}$ & $-1.06 \times 10^{-4}$ \\
$\mathcal{C}_{\phi d}$ & $6.26 \times 10^{-5}$ & 0 & 0 & 0 & 0 \\
$\mathcal{C}_{l l}$ & $-2.79 \times 10^{-3}$ & $-5.6 \times 10^{-4}$ & $2.57 \times 10^{-5}$ & $2.26 \times 10^{-3}$ & $9.4 \times 10^{-4}$ \\
$\mathcal{C}_{l q}^{(3)}$ & $8.9 \times 10^{-4}$ & 0 & 0 & 0 & 0 \\
\hline \hline
\end{tabular}

\section{APPENDIX C: ANALYTICAL EXPRESSIONS OF $\mathcal{F}_{g}$ AND $\mathcal{F}_{p}$}

Here, we report the explicit results for the coefficients $\mathcal{F}_{g}$ and $\mathcal{F}_{p}$ introduced in Eq. (32):

$$
\begin{aligned}
\mathcal{F}_{g}= & \frac{1}{16 \pi^{2}} \frac{1}{\Lambda^{2}}\left(\frac{12 \sqrt{4} 2 \sqrt{G_{\mu}} \mathcal{C}_{W} M_{W}^{3}\left(M_{H}^{2}-2 M_{Z}^{2}\right)\left(M_{Z}^{2}-6 M_{W}^{2}\right)}{M_{Z}^{4}}\right. \\
& +\mathcal{C}_{\phi \square}\left(-6 M_{H}^{2}-12 M_{t}^{2}+9 M_{W}^{2}-\frac{2 M_{Z}^{2}}{3}\right)+\frac{1}{12} \mathcal{C}_{\phi D}\left(-9 M_{H}^{2}-36 M_{t}^{2}+8 M_{W}^{2}-35 M_{Z}^{2}\right) \\
& +\frac{\mathcal{C}_{\phi W} M_{W}^{2}\left(M_{H}^{2}-2 M_{Z}^{2}\right)\left(9 M_{H}^{2}+18 M_{t}^{2}-56 M_{W}^{2}+3 M_{Z}^{2}\right)}{3 M_{Z}^{4}} \\
& +\frac{\mathcal{C}_{\phi B}\left(M_{H}^{2}-2 M_{Z}^{2}\right)\left(M_{Z}^{2}-M_{W}^{2}\right)\left(9 M_{H}^{2}+18 M_{t}^{2}-100 M_{W}^{2}+85 M_{Z}^{2}\right)}{3 M_{Z}^{4}} \\
& +\frac{\mathcal{C}_{\phi W B} M_{W}\left(M_{H}^{2}-2 M_{Z}^{2}\right) \sqrt{M_{Z}^{2}-M_{W}^{2}}\left(3 M_{H}^{2}+18 M_{t}^{2}-42 M_{W}^{2}+56 M_{Z}^{2}\right)}{3 M_{Z}^{4}} \\
& +\frac{2 \sqrt{2} \mathcal{C}_{u W} M_{t} M_{W}\left(2 M_{Z}^{2}-M_{H}^{2}\right)\left(8 M_{W}^{2}-5 M_{Z}^{2}\right)}{M_{Z}^{4}} \\
& +\frac{2 \sqrt{2} \mathcal{C}_{u B} M_{t}\left(M_{H}^{2}-2 M_{Z}^{2}\right)\left(8 M_{W}^{2}-5 M_{Z}^{2}\right) \sqrt{M_{Z}^{2}-M_{W}^{2}}}{M_{Z}^{4}} \\
& +\mathcal{C}_{\phi l}^{(1)}\left(8 M_{Z}^{2}-8 M_{W}^{2}\right)+\mathcal{C}_{\phi l}^{(3)}\left(6 M_{t}^{2}-20 M_{W}^{2}\right)+\mathcal{C}_{\phi e}\left(8 M_{Z}^{2}-8 M_{W}^{2}\right) \\
& +\mathcal{C}_{\phi q}^{(1)}\left(-12 M_{t}^{2}+8 M_{W}^{2}-8 M_{Z}^{2}\right)+6 \mathcal{C}_{\phi q}^{(3)}\left(3 M_{t}^{2}-4 M_{W}^{2}\right)+4 \mathcal{C}_{\phi u}\left(3 M_{t}^{2}+4 M_{W}^{2}-4 M_{Z}^{2}\right) \\
& \left.+\mathcal{C}_{\phi d}\left(8 M_{Z}^{2}-8 M_{W}^{2}\right)-3 \mathcal{C}_{l l} M_{Z}^{2}-6 \mathcal{C}_{l q}^{(3)} M_{t}^{2}\right)
\end{aligned}
$$




$$
\begin{aligned}
\mathcal{F}_{p}= & \frac{1}{16 \pi^{2}} \frac{1}{\Lambda^{2}}\left(\mathcal{C}_{\phi \square}\left(-6 M_{H}^{2}-12 M_{t}^{2}+9 M_{W}^{2}-\frac{2 M_{Z}^{2}}{3}\right)+\frac{1}{12} \mathcal{C}_{\phi D}\left(-9 M_{H}^{2}-36 M_{t}^{2}+8 M_{W}^{2}-35 M_{Z}^{2}\right)\right. \\
& +8 \mathcal{C}_{\phi l}^{(1)}\left(M_{Z}^{2}-M_{W}^{2}\right)+\mathcal{C}_{\phi l}^{(3)}\left(6 M_{t}^{2}-20 M_{W}^{2}\right)+8 \mathcal{C}_{\phi e}\left(M_{Z}^{2}-M_{W}^{2}\right) \\
& +\mathcal{C}_{\phi q}^{(1)}\left(-12 M_{t}^{2}+8 M_{W}^{2}-8 M_{Z}^{2}\right)+6 \mathcal{C}_{\phi q}^{(3)}\left(3 M_{t}^{2}-4 M_{W}^{2}\right)+4 \mathcal{C}_{\phi u}\left(3 M_{t}^{2}+4 M_{W}^{2}-4 M_{Z}^{2}\right) \\
& \left.+8 \mathcal{C}_{\phi d}\left(M_{Z}^{2}-M_{W}^{2}\right)-3 \mathcal{C}_{l l} M_{Z}^{2}-6 \mathcal{C}_{l q}^{(3)} M_{t}^{2}\right)
\end{aligned}
$$

\section{APPENDIX D: ANALYTICAL EXPRESSION FOR $\Delta r$}

Here, we report our result for $\Delta r$ in $R_{\xi}$ gauge,

$$
16 \pi^{2} \Delta r=\frac{\sqrt{2} G_{\mu}}{2} \Delta r_{\mathrm{SM}}+\frac{1}{\Lambda^{2}} \Delta r_{\mathrm{EFT}}
$$

where $\Delta r_{\mathrm{SM}}$ and $\Delta r_{\mathrm{EFT}}$ are

$$
\begin{aligned}
\Delta r_{\mathrm{SM}}= & \left(\frac{6 A_{0}\left(M_{W}^{2}\right)\left(M_{H}^{2}\left(M_{W}^{2}-2 M_{Z}^{2}\right)+M_{W}^{2}\left(3 M_{Z}^{2}-2 M_{W}^{2}\right)\right)}{\left(M_{H}^{2}-M_{W}^{2}\right)\left(M_{W}^{2}-M_{Z}^{2}\right)}+4 A_{0}\left(M_{W}^{2} \xi\right)+\frac{6 M_{W}^{2} A_{0}\left(M_{H}^{2}\right)}{M_{H}^{2}-M_{W}^{2}}\right. \\
& \left.-12 A_{0}\left(M_{t}^{2}\right)+\frac{6 A_{0}\left(M_{Z}^{2}\right)\left(2 M_{W}^{2}-M_{Z}^{2}\right)}{M_{W}^{2}-M_{Z}^{2}}+2 A_{0}\left(M_{Z}^{2} \xi\right)-M_{H}^{2}+6 M_{t}^{2}-2 M_{W}^{2}-M_{Z}^{2}\right)
\end{aligned}
$$

and

$$
\begin{aligned}
\Delta r_{\mathrm{EFT}}= & \frac{1}{\hat{\epsilon}}\left(4 \mathcal{C}_{\phi l}^{(3)}\left(3 M_{t}^{2}-4 M_{W}^{2}\right)+24 \mathcal{C}_{\phi q}^{(3)} M_{W}^{2}+\frac{2}{3} \mathcal{C}_{\phi \square} M_{W}^{2}-6 \mathcal{C}_{l l} M_{Z}^{2}-12 \mathcal{C}_{l q}^{(3)} M_{t}^{2}\right) \\
& +\Delta r_{\mathrm{SM}}\left(\frac{1}{4} \mathcal{C}_{\phi D}-\mathcal{C}_{\phi l}^{(3)}+\frac{1}{2} \mathcal{C}_{l l}\right)+\frac{6 M_{W}^{2}\left(\mathrm{~A}_{0}\left(M_{H}^{2}\right)-\mathrm{A}_{0}\left(M_{W}^{2}\right)\right)-M_{H}^{4}+M_{W}^{4}}{M_{H}^{2}-M_{W}^{2}}\left(\mathcal{C}_{\phi \square}-\frac{1}{2} \mathcal{C}_{\phi D}\right) \\
& +6\left(M_{t}^{2}-2 \mathrm{~A}_{0}\left(M_{t}^{2}\right)\right)\left(\mathcal{C}_{\phi l}^{(3)}+\mathcal{C}_{\phi q}^{(3)}-\mathcal{C}_{l q}^{(3)}-\frac{1}{4} \mathcal{C}_{\phi D}\right)+6 \mathrm{~A}_{0}\left(M_{Z}^{2}\right)\left(\mathcal{C}_{l l}+c_{W}^{2}\left(4 C_{\phi l}^{(3)}-\mathcal{C}_{\phi D}\right)\right) \\
& -12 M_{Z}^{2}\left(c_{W} s_{W} \mathcal{C}_{\phi W B}-\frac{5}{3} c_{W}^{2} \mathcal{C}_{\phi l}^{(3)}-\frac{5}{12} \mathcal{C}_{l l}-\frac{1}{6} \mathcal{C}_{\phi D}\right) \\
& +12\left(\mathrm{~A}_{0}\left(M_{W}^{2}\right)-c_{W}^{2} \mathrm{~A}_{0}\left(M_{Z}^{2}\right)\right)\left(\mathcal{C}_{l l}+\mathcal{C}_{\phi l}^{(3)}+\mathcal{C}_{\phi l}^{(1)}\right) \\
& -\left(\mathrm{A}_{0}\left(\xi M_{W}^{2}\right)+\mathrm{A}_{0}\left(\xi M_{Z}^{2}\right)+2\left(M_{W}^{2}+M_{Z}^{2}\right)\right) \mathcal{C}_{\phi D},
\end{aligned}
$$

and as usual $c_{W}=\frac{M_{W}^{2}}{M_{Z}^{2}}, s_{W}=\sqrt{1-c_{W}^{2}}$, and

$$
A_{0}(x)=-i \frac{(2 \pi \Lambda)^{2 \epsilon}}{\pi^{2}} \int \frac{d^{d} k_{1}}{\left(k_{1}^{2}-x\right)}=\frac{x}{\hat{\epsilon}}+x\left(1-\log \left(\frac{x}{\Lambda^{2}}\right)\right) .
$$

[1] W. Buchmuller and D. Wyler, Effective Lagrangian analysis of new interactions and flavor conservation, Nucl. Phys. B268, 621 (1986).
[2] B. Grzadkowski, M. Iskrzynski, M. Misiak, and J. Rosiek, Dimension-six terms in the standard model Lagrangian, J. High Energy Phys. 10 (2010) 085. 
[3] K. Hagiwara, S. Ishihara, R. Szalapski, and D. Zeppenfeld, Low-energy effects of new interactions in the electroweak boson sector, Phys. Rev. D 48, 2182 (1993).

[4] G. F. Giudice, C. Grojean, A. Pomarol, and R. Rattazzi, The strongly-interacting light Higgs, J. High Energy Phys. 06 (2007) 045.

[5] J. Elias-Miro, J. R. Espinosa, E. Masso, and A. Pomarol, Higgs windows to new physics through $d=6$ operators: constraints and one-loop anomalous dimensions, J. High Energy Phys. 11 (2013) 066.

[6] J. Elias-Miro, J. R. Espinosa, E. Masso, and A. Pomarol, Renormalization of dimension-six operators relevant for the Higgs decays $h \rightarrow \gamma \gamma, \gamma Z$, J. High Energy Phys. 08 (2013) 033.

[7] E. E. Jenkins, A. V. Manohar, and M. Trott, Renormalization group evolution of the standard model dimension six operators I: Formalism and lambda dependence, J. High Energy Phys. 10 (2013) 087.

[8] E. E. Jenkins, A. V. Manohar, and M. Trott, Renormalization Group evolution of the standard model dimension six operators II: Yukawa dependence, J. High Energy Phys. 01 (2014) 035.

[9] R. Alonso, E. E. Jenkins, A. V. Manohar, and M. Trott, Renormalization group evolution of the standard model dimension six operators III: Gauge coupling dependence and phenomenology, J. High Energy Phys. 04 (2014) 159.

[10] R. Gauld, B. D. Pecjak, and D. J. Scott, One-loop corrections to $h \rightarrow b \bar{b}$ and $h \rightarrow \tau \bar{\tau}$ decays in the Standard Model Dimension-6 EFT: four-fermion operators and the large- $m_{t}$ limit, J. High Energy Phys. 05 (2016) 080.

[11] R. Gauld, B. D. Pecjak, and D. J. Scott, QCD radiative corrections for $h \rightarrow b \bar{b}$ in the Standard Model Dimension-6 EFT, Phys. Rev. D 94, 074045 (2016).

[12] C. Hartmann and M. Trott, On one-loop corrections in the standard model effective field theory; the $\Gamma(h \rightarrow \gamma \gamma)$ case, J. High Energy Phys. 07 (2015) 151.

[13] C. Hartmann and M. Trott, Higgs Decay to Two Photons at One Loop in the Standard Model Effective Field Theory, Phys. Rev. Lett. 115, 191801 (2015).

[14] M. Ghezzi, R. Gomez-Ambrosio, G. Passarino, and S. Uccirati, NLO Higgs effective field theory and $\kappa$-framework, J. High Energy Phys. 07 (2015) 175.

[15] A. Falkowski, Effective field theory approach to LHC Higgs data, Pramana 87, 39 (2016).

[16] I. Brivio and M. Trott, Scheming in the SMEFT... and a reparameterization invariance!, J. High Energy Phys. 07 (2017) 148.

[17] A. Dedes, W. Materkowska, M. Paraskevas, J. Rosiek, and K. Suxho, Feynman rules for the standard model effective field theory in $\mathrm{R}_{\xi}$-gauges, J. High Energy Phys. 06 (2017) 143.

[18] B. A. Kniehl, Radiative corrections for $H \rightarrow Z Z$ in the standard model, Nucl. Phys. B352, 1 (1991).

[19] R. N. Cahn, M. S. Chanowitz, and N. Fleishon, Higgs particle production by $Z \rightarrow H$ gamma, Phys. Lett. 82B, 113 (1979).

[20] L. Bergstrom and G. Hulth, Induced Higgs couplings to neutral bosons in $e^{+} e^{-}$collisions, Nucl. Phys. B259, 137 (1985); Erratum, B276, 744 (1986).

[21] A. Denner, Techniques for calculation of electroweak radiative corrections at the one loop level and results for W physics at LEP-200, Fortschr. Phys. 41, 307 (1993).
[22] J. Fleischer and F. Jegerlehner, Radiative corrections to Higgs decays in the extended Weinberg-Salam model, Phys. Rev. D 23, 2001 (1981).

[23] C.-Y. Chen, S. Dawson, and C. Zhang, Electroweak effective operators and Higgs physics, Phys. Rev. D 89, 015016 (2014).

[24] T. Hahn, Generating Feynman diagrams and amplitudes with FeynArts 3, Comput. Phys. Commun. 140, 418 (2001).

[25] A. Alloul, N. D. Christensen, C. Degrande, C. Duhr, and B. Fuks, FeynRules 2.0-A complete toolbox for tree-level phenomenology, Comput. Phys. Commun. 185, 2250 (2014).

[26] G. Passarino and M. J. G. Veltman, One loop corrections for $e^{+} e^{-}$annihilation into mu+ mu- in the Weinberg model, Nucl. Phys. B160, 151 (1979).

[27] R. Mertig, M. Bohm, and A. Denner, FEYN CALC: Computer algebraic calculation of Feynman amplitudes, Comput. Phys. Commun. 64, 345 (1991).

[28] V. Shtabovenko, R. Mertig, and F. Orellana, New developments in FeynCalc 9.0, Comput. Phys. Commun. 207, 432 (2016).

[29] T. Hahn, Automatic loop calculations with FeynArts, FormCalc, and LoopTools, Nucl. Phys. B, Proc. Suppl. 89, 231 (2000).

[30] R. Contino, M. Ghezzi, C. Grojean, M. Muhlleitner, and M. Spira, Effective Lagrangian for a light Higgs-like scalar, J. High Energy Phys. 07 (2013) 035.

[31] B. Grinstein, C. W. Murphy, and D. Pirtskhalava, Searching for new physics in the three-body decays of the Higgs-like particle, J. High Energy Phys. 10 (2013) 077.

[32] G. Isidori, A. V. Manohar, and M. Trott, Probing the nature of the Higgs-like boson via $h \rightarrow V \mathcal{F}$ decays, Phys. Lett. B 728, 131 (2014).

[33] S. Boselli, C. M. Carloni Calame, G. Montagna, O. Nicrosini, F. Piccinini, and A. Shivaji, Higgs decay into four charged leptons in the presence of dimension-six operators, J. High Energy Phys. 01 (2018) 096.

[34] L. Berthier, M. Bjørn, and M. Trott, Incorporating doubly resonant $W^{ \pm}$data in a global fit of SMEFT parameters to lift flat directions, J. High Energy Phys. 09 (2016) 157.

[35] A. Bredenstein, A. Denner, S. Dittmaier, and M. M. Weber, Radiative corrections to the semileptonic and hadronic Higgs-boson decays $\mathrm{H} \rightarrow \mathrm{WW} / \mathrm{ZZ} \rightarrow 4$ fermions, J. High Energy Phys. 02 (2007) 080.

[36] A. Bredenstein, A. Denner, S. Dittmaier, and M. M. Weber, Precision calculations for the Higgs decays $\mathrm{H} \rightarrow \mathrm{ZZ} / \mathrm{WW} \rightarrow$ 4 leptons, Nucl. Phys. B, Proc. Suppl. 160, 131 (2006).

[37] J. F. Gunion, H. E. Haber, G. L. Kane, and S. Dawson, The Higgs hunter's guide, Front. Phys. 80, 1 (2000).

[38] See Supplemental Material at http://link.aps.org/ supplemental/10.1103/PhysRevD.97.093003 for the analytical expressions of the decay amplitudes $H \rightarrow Z Z$ and $H \rightarrow$ $Z \gamma$ in the SMEFT. The results are provided as text files.

[39] I. Brivio and M. Trott, The standard model as an effective field theory, arXiv:1706.08945.

[40] G. Buchalla, O. Cata, and G. D'Ambrosio, Nonstandard Higgs couplings from angular distributions in $h \rightarrow Z \ell^{+} \ell^{-}$, Eur. Phys. J. C 74, 2798 (2014). 